\title{
Vibration Characteristics Analysis of Cylindrical Shell-Plate Coupled Structure Using an Improved Fourier Series Method
}

\author{
Yipeng Cao $₫{ }^{1},{ }^{1}$ Runze Zhang $\left(\mathbb{D},{ }^{1}\right.$ Wenping Zhang, ${ }^{1}$ and Jinzhao Wang ${ }^{2}$ \\ ${ }^{1}$ College of Power and Energy Engineering, Harbin Engineering University, Harbin, China \\ ${ }^{2}$ Luoyang Sunrui Rubber \& Plastic Technology Co., Ltd., Luoyang, China \\ Correspondence should be addressed to Runze Zhang; runze2007625@hrbeu.edu.cn
}

Received 8 June 2017; Accepted 19 November 2017; Published 12 February 2018

Academic Editor: Lorenzo Dozio

Copyright (c) 2018 Yipeng Cao et al. This is an open access article distributed under the Creative Commons Attribution License, which permits unrestricted use, distribution, and reproduction in any medium, provided the original work is properly cited.

\begin{abstract}
A simple yet accurate solution procedure based on the improved Fourier series method (IFSM) is applied to the vibration characteristics analysis of a cylindrical shell-circular plate $(S-P)$ coupled structure subjected to various boundary conditions. By applying four types of coupling springs with arbitrary stiffness at the junction of the coupled structure, the mechanical coupling effects are completely considered. Each of the plate and shell displacement functions is expressed as the superposition of a twodimensional Fourier series and several supplementary functions. The unknown series-expansion coefficients are treated as the generalized coordinates and determined using the familiar Rayleigh-Ritz procedure. Using the IFSM, a unified solution for the $S$ - $P$ coupled structure with symmetrical and asymmetrical boundary conditions can be derived directly without the need to change either the equations of motion or the expressions of the displacements. This solution can be verified by comparing the current results with those calculated by the finite-element method (FEM). The effects of several significant factors, including the restraint stiffness, the coupling stiffness, and the situation of coupling, are presented. The forced vibration behaviors of the $S$ - $P$ coupled structure are also illustrated.
\end{abstract}

\section{Introduction}

The cylindrical shell-circular plate $(S-P)$ coupled structure is widely used as a structural component in various engineering fields, such as naval vessel, spacecraft, and civil building construction. Therefore, a complete understanding of the vibration characteristics of the $S-P$ coupled structure is necessary and of great significance. In the past several decades, many studies [1-5] have been published on cylindrical shells, stiffened shells, plates, and other structures. Based on these dynamic models, a simulation method that can calculate the response of a cylindrical shell-plate combined structure was developed.

Tavakoli and Singh [6] presented a state-space method to analyse the free vibration of a hermetic shell composed of a circular cylinder with two circular end plates, and the analytical results were compared with model measurements. Cheng and Nicolas [7] studied the inherent characteristics of a circular cylindrical shell closed at one end by a circular plate with various boundary supports. However, they neglect the in-plane motion of the circular plate. Huang and Soedel [8] used the receptance method to solve the $S$ - $P$ structure with shear diaphragm-shear diaphragm boundary conditions. Tso and Hansen [9] studied the wave propagation through cylinder/plate junctions and provided a theoretical basis for the study of the bulkhead. Chen et al. [10] studied the free vibration of a ring-stiffened cylindrical shell with intermediate large frame ribs, and the equations of motion of annular circular plates were used to describe the motions of the ribs.

In the analyses cited above, the focus was the free vibration of a circular cylindrical shell coupled with end plates; little literature $[11,12]$ is available on the free and forced vibration of the $S$ - $P$ coupled structure in which the circular plate connects the shell in the middle. In this paper, a unified dynamical model for the $S$ - $P$ coupled structure with arbitrary boundary conditions is presented. To eliminate the potential discontinuities and accelerate the convergence of the displacement functions, the displacements of the cylindrical shell and the circular plate are expressed as an improved Fourier series. Translational and rotational 


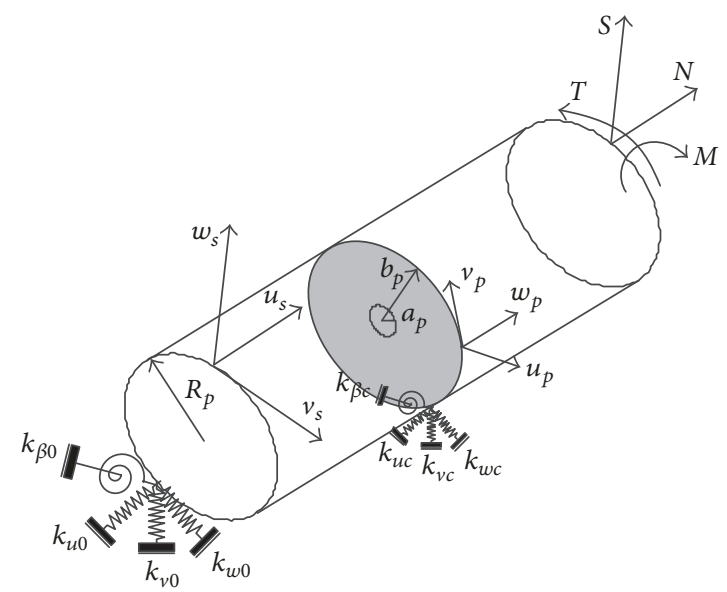

Figure 1: Coordinate system of the $S$ - $P$ coupled structure.

springs with independent stiffness are introduced to simulate the complex boundary and coupling conditions. Finally, an analytical method is presented to analyse the free and forced vibrations of the $S-P$ coupled structure. To validate the proposed method, selected results for symmetrical and asymmetrical boundary conditions are compared with those calculated by the finite-element method (FEM). Furthermore, the effects of the restraint stiffness, the coupling stiffness, and the coupling situation are discussed in detail. The forced vibration behavior is also presented.

\section{Theoretical Formulations}

2.1. Theoretical Model. An S-P coupled structure is schematically presented in Figure 1 . The cylindrical shell is described with the $(r, \theta, x)$ cylindrical coordinate system, in which $x_{s}, r_{s}$, and $\theta_{s}$ denote the axial, circumferential, and radial directions, respectively. The displacements of the cylindrical shell with respect to this coordinate system are denoted by $u_{s}$, $v_{s}$, and $w_{s}$ in the $x_{s}, \theta_{s}$, and $r_{s}$ directions, respectively. $L_{s}, h_{s}$, and $R_{s}$ are the length, thickness, and middle-surface radius of the shells, respectively. $E_{s}, \mu_{s}$, and $\rho_{s}$ are Young's modulus of elasticity, Poisson's ratio, and density, respectively. For the plate, an annular plate model is used in the coupled system as a basic structural component that can be used to model the annular and circular plates.

For the circular plate, the equations of strain energy appear to become singular at the pole of the coordinate system. However, it has been observed that the existence of the singularity has a negligible effect on the physical response of the plate. Therefore, the method adopted here is to assume that the inner radius of the annular plate approaches 0 , which avoids the coordinate-dependent singularity.

To simulate the arbitrary elastic boundary and the coupling conditions, an artificial spring technique is adopted here. According to the boundary conditions, four springs distributed along the boundary are used to match the bending moments $M$, transverse shear $S$, tangential shear force $T$, and axial force $N$ separately. The different boundary and coupling conditions can be easily realized by changing the stiffness of the corresponding springs. At the left end of the cylindrical shell, $k_{u 0}, k_{v 0}$, and $k_{w 0}$ denote the linear springs in the $x, \theta$, and $r$ directions, respectively, and $k_{\beta 0}$ denotes the rotational spring stiffness around the $\theta$ direction. Similarly, a set of springs $k_{u 0}, k_{v 0}, k_{w 0}$, and $k_{\beta 0}$ can also be applied to the right side.

2.2. Theoretical Formulations. The displacement admissible functions of the cylindrical shell and circular plate can be expediently expressed by an improved Fourier series composed of the standard Fourier cosine series and supplementary functions

$$
\begin{aligned}
& u_{s}(x, \theta, t) \\
& =\left\{\sum_{n=0}^{N}\left(\sum_{m=0}^{M} A_{m n}^{s} \cos \left(\lambda_{m}^{s} x\right)+\sum_{l=1}^{2} a_{l}^{s} \xi_{l}(x)\right) \cos (n \theta)\right. \\
& \left.+\sum_{n=1}^{N}\left(\sum_{m=0}^{M} \widetilde{A}_{m n}^{s} \cos \left(\lambda_{m}^{s} x\right)+\sum_{l=1}^{2} \widetilde{a}_{l}^{s} \xi_{l}(x)\right) \sin (n \theta)\right\} \\
& \cdot e^{j \omega t} \text {, } \\
& v_{s}(x, \theta, t) \\
& =\left\{\sum_{n=1}^{N}\left(\sum_{m=0}^{M} B_{m n}^{s} \cos \left(\lambda_{m}^{s} x\right)+\sum_{l=1}^{2} b_{l}^{s} \xi_{l}(x) \sin (n \theta)\right)\right. \\
& \left.+\sum_{n=0}^{N}\left(\sum_{m=0}^{M} \widetilde{B}_{m n}^{S} \cos \left(\lambda_{m}^{s} x\right)+\sum_{l=1}^{2} \widetilde{b}_{l}^{s} \xi_{l}(x)\right) \cos (n \theta)\right\} \\
& \text { - } e^{j \omega t} \text {, } \\
& w_{s}(x, \theta, t) \\
& =\left\{\sum_{n=0}^{N}\left(\sum_{m=0}^{M} C_{m n}^{s} \cos \left(\lambda_{m}^{s} x\right)+\sum_{l=1}^{4} c_{l}^{s} \xi_{l}(x)\right) \cos (n \theta)\right. \\
& \left.+\sum_{n=1}^{N}\left(\sum_{m=0}^{M} \widetilde{C}_{m n}^{s} \cos \left(\lambda_{m}^{s} x\right)+\sum_{l=1}^{4} \widetilde{\mathcal{c}}_{l}^{s} \xi_{l}(x)\right) \sin (n \theta)\right\} \\
& \cdot e^{j \omega t}
\end{aligned}
$$




$$
\begin{aligned}
u_{p}(s, \theta, t) & \\
= & \left\{\sum_{n=0}^{N}\left(\sum_{m=0}^{M} A_{m n}^{p} \cos \left(\lambda_{m}^{p} s\right)+\sum_{l=1}^{2} a_{l}^{p} \xi_{l}(s)\right) \cos (n \theta)\right. \\
& \left.+\sum_{n=1}^{N}\left(\sum_{m=0}^{M} \widetilde{A}_{m n}^{p} \cos \left(\lambda_{m}^{p} s\right)+\sum_{l=1}^{2} \widetilde{a}_{l}^{p} \xi_{l}(s)\right) \sin (n \theta)\right\} \\
& \cdot e^{j \omega t}, \\
v_{p}(s, \theta, t) & \\
= & \left\{\sum_{n=1}^{N}\left(\sum_{m=0}^{M} B_{m n}^{p} \cos \left(\lambda_{m}^{p} s\right)+\sum_{l=1}^{2} b_{l}^{p} \xi_{l}(s)\right) \sin (n \theta)\right. \\
& \left.+\sum_{n=0}^{N}\left(\sum_{m=0}^{M} \widetilde{B}_{m n}^{p} \cos \left(\lambda_{m}^{p} s\right)+\sum_{l=1}^{2} \widetilde{b}_{l}^{p} \xi_{l}(s)\right) \cos (n \theta)\right\} \\
& \cdot e^{j \omega t}, \\
w_{p} & (s, \theta, t) \\
& =\left\{\sum_{n=0}^{N}\left(\sum_{m=0}^{M} C_{m n}^{p} \cos \left(\lambda_{m}^{p} s\right)+\sum_{l=1}^{4} c_{l}^{p} \xi_{l}(s)\right) \cos (n \theta)\right. \\
& \left.+\sum_{n=1}^{N}\left(\sum_{m=0}^{M} \widetilde{C}_{m n}^{p} \cos \left(\lambda_{m}^{p} s\right)+\sum_{l=1}^{4} \widetilde{c}_{l}^{p} \xi_{l}(s)\right) \sin (n \theta)\right\} \\
& +e^{j \omega t},
\end{aligned}
$$

where $\omega$ denotes the angular frequency and $t$ is the time variable. $\lambda_{m}^{s}=(m \pi) / L,(m=0,1,2, \ldots, M), m$ and $n$ are the sequence number of the expansions in the axial and circumferential directions. $q=\left[A_{m n}^{i}, a_{l}^{i}, \widetilde{A}_{m n}^{i}, \widetilde{a}_{l}^{i}, B_{m n}^{i}, b_{l}^{i}\right.$, $\left.\widetilde{B}_{m n}^{i}, \widetilde{b}_{l}^{i}, C_{m n}^{i}, c_{l}^{i}, \widetilde{C}_{m n}^{i}, \widetilde{c}_{l}^{i}\right](i=S, P)$ are the coefficients of the series expansions. The introduction of the supplementary functions $\xi_{l}(x)(l=1,2,3,4)$ can not only remove the potential discontinuities at the joint and boundary but also ensure and accelerate the convergence of expansion series. The supplementary functions $\xi_{l}(x)$ can be any continuous closed form functions and allow easily exact differential and integral calculus for high accuracy. The supplementary functions $\xi_{l}(s)(l=1,2,3,4)$ for the circular plate can be obtained by replacing $x$ with $s$. A set of auxiliary functions describing the cylindrical shell is selected as follows [13]:

$$
\left\{\begin{array}{l}
\xi_{1}(x) \\
\xi_{2}(x) \\
\xi_{3}(x) \\
\xi_{4}(x)
\end{array}\right\}=\left\{\begin{array}{c}
\frac{6 L x-2 L^{2}-3 x^{2}}{6 L} \\
\frac{3 x^{2}-L^{2}}{6 L} \\
\frac{-15 x^{4}+60 L x^{3}-60 L^{2} x^{2}+8 L^{4}}{360 L} \\
\frac{15 x^{4}-30 L^{2} x^{2}+7 L^{4}}{360 L}
\end{array}\right\} .
$$

From (2) it can be seen that the highest order of the supplementary function is four. Thus, the second-order derivatives of in-plane displacements $(u$ and $v)$ as well as the third-order derivatives of the radial displacement $(w)$ can be achieved.

Once the form of the solutions has been established for the circular plate and cylindrical shell, the remaining task is to find a suitable set of expansion coefficients that will ensure that the series satisfies the governing equations, boundary conditions, and joint continuity. A solution can be obtained either in strong form by making the series satisfy the relevant equations exactly or in weak form by solving for the series coefficients approximately. The Ritz method is a direct method for finding an approximate solution of a boundary-value problem. Since the constructed solutions are sufficiently smooth over the solution domain, the unknown series coefficients are calculated using the Rayleigh-Ritz technique, which is equivalent to solving the governing equations, boundary conditions, and coupling conditions directly.

The energy expressions are essential for achieving sufficiently accurate solutions. The entire energy for the coupled cylindrical shell-plate system includes five parts: the stain energy $V_{s}$ and the kinetic energy $T_{s}$ for the cylindrical shell, the stain energy $V_{p}$ and the kinetic energy $T_{p}$ for the annular plate, the spring elastic potential energy $V_{b}$ denoting the energy caused by boundary conditions at the ends of the shell, the potential energy $V_{c}$ stored at the junction between adjacent substructures, and the energy function $V_{f}$ caused by the external loads.

Adopting Reissner's thin shell theory, the strain energy $V_{s}$ and the kinetic energy $T_{s}$ for the cylindrical shell can be written as

$$
\begin{aligned}
V_{s} & =\frac{E_{s} h_{s}}{2\left(1-\mu^{2}\right)} \int_{0}^{2 \pi} \int_{0}^{l}\left\{\left(\frac{\partial u}{\partial x}+\frac{\partial v}{R \partial \theta}+\frac{w}{R}\right)^{2}\right. \\
& -2(1-\mu) \frac{\partial u}{\partial x}\left(\frac{\partial v}{R \partial \theta}+\frac{w}{R}\right) \\
& \left.+\frac{(1-\mu)}{2}\left(\frac{\partial v}{\partial x}+\frac{\partial u}{R \partial \theta}\right)^{2}\right\} R d x d \theta \\
& +\frac{E_{s} h_{s}{ }^{3}}{24\left(1-\mu^{2}\right)} \int_{0}^{2 \pi} \int_{0}^{l}\left\{\left(\frac{\partial^{2} w}{\partial x^{2}}+\frac{\partial^{2} w}{R^{2} \partial \theta^{2}}\right)^{2}\right. \\
& \left.-2(1-\mu)\left[\frac{\partial^{2} w}{\partial x^{2}} \frac{\partial^{2} w}{R^{2} \partial \theta^{2}}-\left(\frac{\partial^{2} w}{R \partial x \partial \theta}\right)^{2}\right]\right\} R_{s} d x d \theta \\
& +\frac{E_{s} h_{s}{ }^{3}}{24 R^{2}\left(1-\mu^{2}\right)} \int_{0}^{2 \pi} \int_{0}^{L}\left\{-2 \mu \frac{\partial v}{\partial \theta} \frac{\partial^{2} w}{\partial x^{2}}-2 \frac{\partial v}{\partial \theta} \frac{\partial^{2} w}{R^{2} \partial \theta^{2}}\right. \\
& +\left(\frac{\partial v}{R \partial \theta}\right)^{2}-4(1-\mu) \frac{\partial v}{\partial x} \frac{\partial^{2} w}{\partial x \partial \theta} \\
& \left.+2(1-\mu)\left(\frac{\partial v}{\partial x}\right)^{2}\right\} R_{s} d x d \theta,
\end{aligned}
$$




$$
T_{s}=\int_{0}^{L} \int_{0}^{2 \pi}\left\{\frac{\rho_{s} h_{s}}{2}\left[\left(\frac{\partial u}{\partial t}\right)^{2}+\left(\frac{\partial v}{\partial t}\right)^{2}+\left(\frac{\partial w}{\partial t}\right)^{2}\right]\right\}
$$$$
\cdot R_{s} d \theta d x \text {. }
$$

According to the thin plate theory (Leissa, 1993), the strain energy and the kinetic energy for the circular plate can be written as

$$
\begin{aligned}
V_{p} & =\frac{E_{p} h_{p}}{2\left(1-\mu^{2}\right)} \int_{0}^{2 \pi} \int_{0}^{R_{p}}\left\{\left(\varepsilon_{s, p}\right)^{2}+\left(\varepsilon_{\theta, p}\right)^{2}+2 \mu \varepsilon_{s, p} \varepsilon_{\theta, p}\right. \\
& \left.+\frac{(1-\mu)}{2}\left(\gamma_{s \theta, p}\right)^{2}\right\}(s+a) d s d \theta \\
& +\frac{E_{p} h_{p}^{3}}{24\left(1-\mu^{2}\right)} \int_{0}^{2 \pi} \int_{0}^{R_{p}}\left\{\left(k_{s, p}\right)^{2}+\left(k_{\theta, p}\right)^{2}\right. \\
& \left.+2 \mu k_{s, p} k_{\theta, p}+\frac{(1-\mu)}{2}\left(\tau_{s \theta, p}\right)^{2}\right\}(s+a) d s d \theta, \\
T_{p} & =\frac{\rho_{p} h_{p}}{2} \\
& \cdot \int_{0}^{L} \int_{0}^{2 \pi}\left\{\left[\left(\frac{\partial u_{p}}{\partial t}\right)^{2}+\left(\frac{\partial v_{p}}{\partial t}\right)^{2}+\left(\frac{\partial w_{p}}{\partial t}\right)^{2}\right]\right\}(s \\
& +a) d s d \theta,
\end{aligned}
$$

where $\varepsilon_{s, p}, \varepsilon_{\theta, p}$, and $\gamma_{s \theta, p}$ are the normal and shear strains in the middle surface of the circular plate, $k_{s, p}$ and $k_{\theta, p}$ are the mid-surface changes in curvature, and $\tau_{s \theta, p}$ is the mid-surface twist. The strain-displacement equations can be written as

$$
\begin{aligned}
\varepsilon_{s, p} & =\frac{\partial u_{p}}{\partial s}, \\
\varepsilon_{\theta, p} & =\frac{1}{s+a}\left(\frac{\partial v_{p}}{\partial s}+u_{p}\right), \\
\gamma_{s \theta, p} & =\frac{\partial v_{p}}{\partial s}+\frac{1}{s+a}\left(\frac{\partial u_{p}}{\partial s}-v_{p}\right), \\
k_{s, p} & =-\frac{\partial^{2} w_{p}}{\partial s^{2}}, \\
k_{\theta, p} & =-\frac{1}{(s+a)^{2}} \frac{\partial^{2} w_{p}}{\partial s^{2}}-\frac{1}{s+a} \frac{\partial w_{p}}{\partial s}, \\
\tau_{s \theta, p} & =-\frac{2}{s+a} \frac{\partial^{2} w_{p}}{\partial s \partial \theta}+\frac{1}{(s+a)^{2}} \frac{\partial w_{p}}{\partial s} .
\end{aligned}
$$

Consider displacement continuity conditions at the junction and boundary conditions of the coupled system; the potential energy stored in the boundary and coupling springs can be written as

$$
V_{c}=\frac{1}{2} \int_{0}^{2 \pi}\left[k_{c u}\left(u_{s}-w_{p}\right)^{2}+k_{c v}\left(v_{s}-v_{p}\right)^{2}\right.
$$

$$
\left.+k_{c w}\left(w_{s}-u_{p}\right)^{2}+k_{c \beta}\left(\frac{\partial w_{s}}{\partial x}-\frac{\partial w_{p}}{\partial r}\right)^{2}\right]\left.\right|_{x=x_{c}, s=b}
$$

- $R_{s} d \theta$

$$
\begin{aligned}
V_{b} & =\frac{1}{2} \int_{0}^{2 \pi}\left[k_{u 0} u_{s}^{2}+k_{v 0} v_{s}^{2}+k_{w 0} w_{s}^{2}\right. \\
& \left.+k_{\beta 0}\left(\frac{\partial w_{s}}{\partial x}\right)^{2}\right]_{x=0} R_{s} d \theta+\frac{1}{2} \int_{0}^{2 \pi}\left[k_{u 1} u_{s}^{2}+k_{v 1} v_{s}^{2}\right. \\
& \left.+k_{w 1} w_{s}^{2}+k_{\beta 1}\left(\frac{\partial w_{s}}{\partial x}\right)^{2}\right]_{x=L} R_{s} d \theta
\end{aligned}
$$

The frequency response function (FRF) of the coupled structure can be calculated considering the potential energy $V_{f}$ caused by an external point loads. Under the application of the point force located at $\left(x_{0}, \theta_{0}\right)$ the potential energy $V_{f}$ can be written as

$$
\begin{aligned}
V_{f} & =\int_{0}^{2 \pi} \int_{0}^{L}\left(f_{u i} u_{i}+f_{v i} v_{i}+f_{w i} w_{i}\right) \delta\left(x-x_{0}, \theta-\theta_{0}\right) \\
& \cdot R\left(x_{i}\right) d x_{i} d \theta_{i},
\end{aligned}
$$

where $f_{u i}, f_{v i}$, and $f_{w i}$ are the external force in the $x_{s}, \theta_{s}$, and $r_{s}$ directions, respectively. $i=s, p$ denote the cases of the external force acting on the cylindrical shell or circular plate, respectively. $\delta$ denotes the Dirac function.

When all of the energy expressions are prepared, the Rayleigh-Ritz technique will be used to obtain a weak form of solutions. Thus the Lagrangian energy function can be written as

$$
L=\sum_{i=1}^{N_{s}}\left(V_{s}+T_{s}\right)+\sum_{i=1}^{N_{p}}\left(V_{p}+T_{p}\right)+V_{c}+V_{b}+V_{f}
$$

where $N_{s}$ and $N_{p}$ are the number of the cylindrical shells and circular plates in the coupling structure. The current solution procedure can be utilized to derive the characteristic equation of the $S$ - $P$ coupled structure with more substructures readily. Substituting the total energy into (8), the eigenvalue problem is formulated by minimizing the Lagrangian function with respect to the arbitrary coefficients. This corresponds to the following equation:

$$
\frac{\partial L}{\partial \mathbf{q}}=0
$$

where $\mathbf{q}$ denotes the coefficient vector of the series expansions. Equation (9) yields a set of liner and homogeneous algebraic equations in the unknown coefficients. Then the final system equation can be obtained and summarized in a matrix form as

$$
\left(\mathbf{K}-\omega^{2} \mathbf{M}\right) \mathbf{q}=\mathbf{F}
$$


TABLE 1: The frequency for the coupled structure with symmetrical boundary condition $f / \mathrm{Hz}$.

\begin{tabular}{|c|c|c|c|c|c|c|c|c|c|}
\hline \multirow{2}{*}{ Mode } & \multicolumn{3}{|c|}{ F-F } & \multicolumn{3}{|c|}{ S-S } & \multicolumn{3}{|c|}{$\mathrm{C}-\mathrm{C}$} \\
\hline & Current & Ansys & Errors & Current & Ansys & Errors & Current & Ansys & Errors \\
\hline (1) & 25.01 & 25.03 & 0.07 & 88.21 & 87.64 & 0.65 & 85.99 & 85.42 & 0.67 \\
\hline (2) & 25.01 & 25.03 & 0.06 & 179.04 & 177.85 & 0.67 & 179.05 & 177.85 & 0.67 \\
\hline (3) & 68.07 & 68.13 & 0.09 & 179.04 & 177.85 & 0.67 & 179.05 & 177.85 & 0.67 \\
\hline (4) & 68.07 & 68.13 & 0.09 & 293.83 & 291.74 & 0.72 & 293.84 & 291.74 & 0.72 \\
\hline (5) & 88.21 & 87.64 & 0.65 & 293.83 & 291.88 & 0.67 & 293.84 & 291.88 & 0.67 \\
\hline (6) & 128.55 & 128.69 & 0.11 & 295.67 & 295.76 & 0.03 & 335.01 & 332.70 & 0.69 \\
\hline (7) & 128.55 & 128.69 & 0.11 & 295.67 & 295.76 & 0.03 & 356.56 & 356.70 & 0.04 \\
\hline (8) & 170.33 & 170.33 & 0.00 & 329.87 & 329.98 & 0.03 & 356.56 & 356.70 & 0.04 \\
\hline (9) & 170.33 & 170.33 & 0.00 & 329.87 & 329.98 & 0.03 & 376.51 & 376.64 & 0.03 \\
\hline (10) & 179.37 & 178.18 & 0.67 & 337.94 & 335.63 & 0.69 & 376.51 & 376.64 & 0.03 \\
\hline (11) & 179.37 & 178.18 & 0.67 & 344.03 & 344.17 & 0.04 & 417.62 & 417.27 & 0.08 \\
\hline (12) & 195.77 & 195.26 & 0.26 & 344.03 & 344.17 & 0.04 & 417.62 & 417.27 & 0.08 \\
\hline (13) & 195.77 & 195.26 & 0.26 & 354.59 & 354.00 & 0.17 & 423.03 & 421.88 & 0.27 \\
\hline (14) & 206.36 & 206.65 & 0.14 & 354.59 & 354.00 & 0.17 & 423.03 & 421.88 & 0.27 \\
\hline (15) & 206.36 & 206.65 & 0.14 & 375.55 & 375.48 & 0.02 & 424.65 & 424.86 & 0.05 \\
\hline (16) & 219.70 & 219.98 & 0.13 & 375.55 & 375.48 & 0.02 & 424.65 & 424.86 & 0.05 \\
\hline (17) & 219.70 & 219.98 & 0.13 & 417.24 & 415.28 & 0.47 & 430.00 & 426.90 & 0.73 \\
\hline (18) & 293.84 & 291.75 & 0.72 & 417.24 & 415.28 & 0.47 & 430.00 & 426.90 & 0.73 \\
\hline (19) & 293.84 & 291.88 & 0.67 & 429.95 & 426.86 & 0.72 & 455.71 & 455.92 & 0.05 \\
\hline (20) & 301.50 & 302.00 & 0.17 & 429.95 & 426.86 & 0.72 & 455.71 & 455.92 & 0.05 \\
\hline
\end{tabular}

where $\mathbf{K}$ and $\mathbf{M}$ are the stiffness and mass matrices of the coupling structure, respectively. $\mathbf{F}$ represents the external force vector. They are written as

$$
\begin{aligned}
& \mathbf{K}=\left[\begin{array}{cc}
\mathbf{K}_{\mathrm{s}} & \mathbf{K}_{\mathrm{sp}} \\
\mathbf{K}_{\mathrm{sp}}^{\mathrm{T}} & \mathbf{K}_{\mathbf{p}}
\end{array}\right], \\
& \mathbf{M}=\left[\begin{array}{ll}
\mathbf{M}_{\mathbf{s}} & \\
& \mathbf{M}_{\mathbf{p}}
\end{array}\right] .
\end{aligned}
$$

\section{Numerical Example and Discussions}

In this section, several examples are presented to evaluate the accuracy and reliability of the proposed method. First, symmetrical and asymmetric boundary conditions are given for the $S$ - $P$ coupled structure. The excellent accuracy of the presented solution is validated through comparison with the results obtained from the FEM. Then, arbitrary positions of the coupling shell are studied. The characteristics of the response are discussed as well.

Unless otherwise specified, the properties for these coupled structures are summarized as follows: length of the shell $L_{s}=1.2 \mathrm{~m}$, thickness $h_{s}=0.002 \mathrm{~m}$, mean radius $R_{s}=$ $0.24 \mathrm{~m}$, outer diameter of circular plate $b_{p}=0.24 \mathrm{~m}$, and interdiameter $a_{p}=10^{-4} b_{p}$. Material properties are as follows: $\rho_{s}=\rho_{p}=7800 \mathrm{~kg} / \mathrm{m}^{3}, E_{s}=E_{p}=2.1 \mathrm{~kg} / \mathrm{m}^{3}$, and $\mu_{s}=\mu_{p}=$ 0.3 . The circular plate is coupled to the middle of shells along the axial direction.
3.1. Validation Study. In this subsection, free vibration results of the $S$ - $P$ coupled structure with symmetrical and asymmetric boundary conditions are studied. For convenience, the symbols F, S, C, and E are introduced and denote free, simplesupport, clamped, and elastic-support boundary conditions, respectively. In Tables 1 and 2, the calculated first twenty natural frequencies are compared with the results obtained using ANSYS. Through this comparison, we find that the discrepancies between the current method and ANSYS are acceptable, with a maximum error of approximately $0.8 \%$. To enhance our understanding of the vibration behaviors of the coupled structures, the first six mode shapes for the structures with various boundary conditions are plotted in Figures 2-7. It is clear that the two sets of modes are essentially identical.

3.2. The Coupled Structure with Arbitrary Coupling Position. In this subsection, selected examples are presented to illustrate the effects of the position of the junction. Various positions of the junction, including the 1/4-, 1/3-, and 1/2-length positions of the shell near the right end, are examined here.

Table 3 shows the comparison of natural frequencies obtained by the proposed method and FEM. Figure 8 shows the first six mode shapes of the $S-P$ coupled structure with various positions of the junction. The frequency of the circular plate does not change significantly; however, the frequency of the cylindrical shell increases as the position of the junction moves from the left end to the middle of the shell.

3.3. The Effect of the Boundary Restraint. In this section, the effects of the boundary spring on the vibration characteristics of the coupled structure are investigated. To understand the 
TABLE 2: The frequency for coupled structure with asymmetrical boundary condition $f / \mathrm{Hz}$.

\begin{tabular}{|c|c|c|c|c|c|c|c|c|c|}
\hline \multirow{2}{*}{ Mode } & \multicolumn{3}{|c|}{ F-F } & \multicolumn{3}{|c|}{ S-S } & \multicolumn{3}{|c|}{$\mathrm{C}-\mathrm{C}$} \\
\hline & Current & Ansys & Errors & Current & Ansys & Errors & Current & Ansys & Errors \\
\hline (1) & 88.21 & 87.64 & 88.21 & 85.98 & 85.41 & 0.67 & 85.98 & 85.41 & 0.67 \\
\hline (2) & 139.26 & 139.07 & 139.26 & 142.58 & 142.32 & 0.18 & 179.04 & 177.85 & 0.67 \\
\hline (3) & 139.26 & 139.07 & 139.26 & 142.58 & 142.32 & 0.18 & 179.04 & 177.85 & 0.67 \\
\hline (4) & 147.90 & 147.99 & 147.90 & 149.75 & 149.82 & 0.05 & 293.84 & 291.74 & 0.72 \\
\hline (5) & 147.90 & 147.99 & 147.90 & 149.75 & 149.82 & 0.05 & 293.84 & 291.88 & 0.67 \\
\hline (6) & 179.12 & 177.93 & 179.12 & 178.98 & 177.79 & 0.67 & 312.95 & 312.98 & 0.01 \\
\hline (7) & 179.12 & 177.93 & 179.12 & 178.98 & 177.79 & 0.67 & 312.95 & 312.98 & 0.01 \\
\hline (8) & 212.16 & 212.44 & 212.16 & 212.81 & 213.10 & 0.13 & 334.81 & 332.51 & 0.69 \\
\hline (9) & 212.16 & 212.44 & 212.16 & 212.81 & 213.10 & 0.13 & 352.81 & 352.93 & 0.03 \\
\hline (10) & 229.81 & 228.79 & 229.81 & 230.42 & 229.33 & 0.47 & 352.81 & 352.93 & 0.03 \\
\hline (11) & 229.81 & 228.79 & 229.81 & 230.42 & 229.33 & 0.47 & 357.38 & 357.23 & 0.04 \\
\hline (12) & 293.84 & 291.76 & 293.84 & 265.27 & 265.19 & 0.03 & 357.38 & 357.23 & 0.04 \\
\hline (13) & 293.84 & 291.89 & 293.84 & 265.27 & 265.19 & 0.03 & 401.76 & 401.00 & 0.19 \\
\hline (14) & 303.71 & 304.22 & 303.71 & 293.85 & 291.76 & 0.72 & 401.76 & 401.00 & 0.19 \\
\hline (15) & 303.71 & 304.22 & 303.71 & 293.85 & 291.89 & 0.67 & 403.79 & 403.61 & 0.04 \\
\hline (16) & 318.17 & 318.15 & 318.17 & 303.93 & 304.45 & 0.17 & 403.79 & 403.61 & 0.04 \\
\hline (17) & 318.17 & 318.15 & 318.17 & 303.93 & 304.45 & 0.17 & 429.98 & 426.88 & 0.73 \\
\hline (18) & 337.94 & 335.63 & 337.94 & 334.81 & 332.51 & 0.69 & 429.98 & 426.88 & 0.73 \\
\hline (19) & 355.63 & 355.75 & 355.63 & 369.09 & 369.22 & 0.04 & 443.39 & 443.63 & 0.05 \\
\hline$(20)$ & 355.63 & 355.75 & 355.63 & 369.09 & 369.22 & 0.04 & 443.39 & 443.63 & 0.05 \\
\hline
\end{tabular}

TABLE 3: The frequency of the coupled structures with different coupling-junction positions $f / \mathrm{Hz}$.

\begin{tabular}{|c|c|c|c|c|c|c|c|c|c|}
\hline \multirow{2}{*}{ Mode } & \multicolumn{3}{|c|}{$1 / 4$} & \multicolumn{3}{|c|}{$1 / 3$} & \multicolumn{3}{|c|}{$1 / 2$} \\
\hline & Current & Ansys & Errors & Current & Ansys & Errors & Current & Ansys & Errors \\
\hline (1) & 88.13 & 87.64 & 0.56 & 88.02 & 87.74 & 0.32 & 88.21 & 87.64 & 0.65 \\
\hline (2) & 178.87 & 177.84 & 0.58 & 178.66 & 178.04 & 0.35 & 179.04 & 177.85 & 0.67 \\
\hline (3) & 178.87 & 177.84 & 0.58 & 178.66 & 178.04 & 0.35 & 179.04 & 177.85 & 0.67 \\
\hline (4) & 219.62 & 219.48 & 0.06 & 250.24 & 250.21 & 0.01 & 293.83 & 291.74 & 0.72 \\
\hline (5) & 219.62 & 219.48 & 0.06 & 250.24 & 250.21 & 0.01 & 293.83 & 291.88 & 0.67 \\
\hline (6) & 241.26 & 241.34 & 0.03 & 255.51 & 255.74 & 0.09 & 295.67 & 295.76 & 0.03 \\
\hline (7) & 241.26 & 241.34 & 0.03 & 255.51 & 255.74 & 0.09 & 295.67 & 295.76 & 0.03 \\
\hline (8) & 288.70 & 288.05 & 0.23 & 293.26 & 292.05 & 0.41 & 329.87 & 329.98 & 0.03 \\
\hline (9) & 288.70 & 288.05 & 0.23 & 293.26 & 292.18 & 0.37 & 329.87 & 329.98 & 0.03 \\
\hline (10) & 293.58 & 291.74 & 0.63 & 323.94 & 324.50 & 0.17 & 337.94 & 335.63 & 0.69 \\
\hline (11) & 293.58 & 291.87 & 0.59 & 323.94 & 324.50 & 0.17 & 344.03 & 344.17 & 0.04 \\
\hline (12) & 317.21 & 317.44 & 0.07 & 337.27 & 335.97 & 0.39 & 344.03 & 344.17 & 0.04 \\
\hline (13) & 317.21 & 317.44 & 0.07 & 344.43 & 344.04 & 0.11 & 354.59 & 354.00 & 0.17 \\
\hline (14) & 337.62 & 335.59 & 0.61 & 344.43 & 344.04 & 0.11 & 354.59 & 354.00 & 0.17 \\
\hline (15) & 419.03 & 419.07 & 0.01 & 427.15 & 427.34 & 0.04 & 375.55 & 375.48 & 0.02 \\
\hline (16) & 419.03 & 419.07 & 0.01 & 427.15 & 427.34 & 0.04 & 375.55 & 375.48 & 0.02 \\
\hline (17) & 423.29 & 423.69 & 0.09 & 429.23 & 428.22 & 0.24 & 417.24 & 415.28 & 0.47 \\
\hline (18) & 423.29 & 423.69 & 0.09 & 429.23 & 428.22 & 0.24 & 417.24 & 415.28 & 0.47 \\
\hline (19) & 429.66 & 426.91 & 0.64 & 449.12 & 449.47 & 0.08 & 429.95 & 426.86 & 0.72 \\
\hline (20) & 429.66 & 426.91 & 0.64 & 449.12 & 449.47 & 0.08 & 429.95 & 426.86 & 0.72 \\
\hline
\end{tabular}



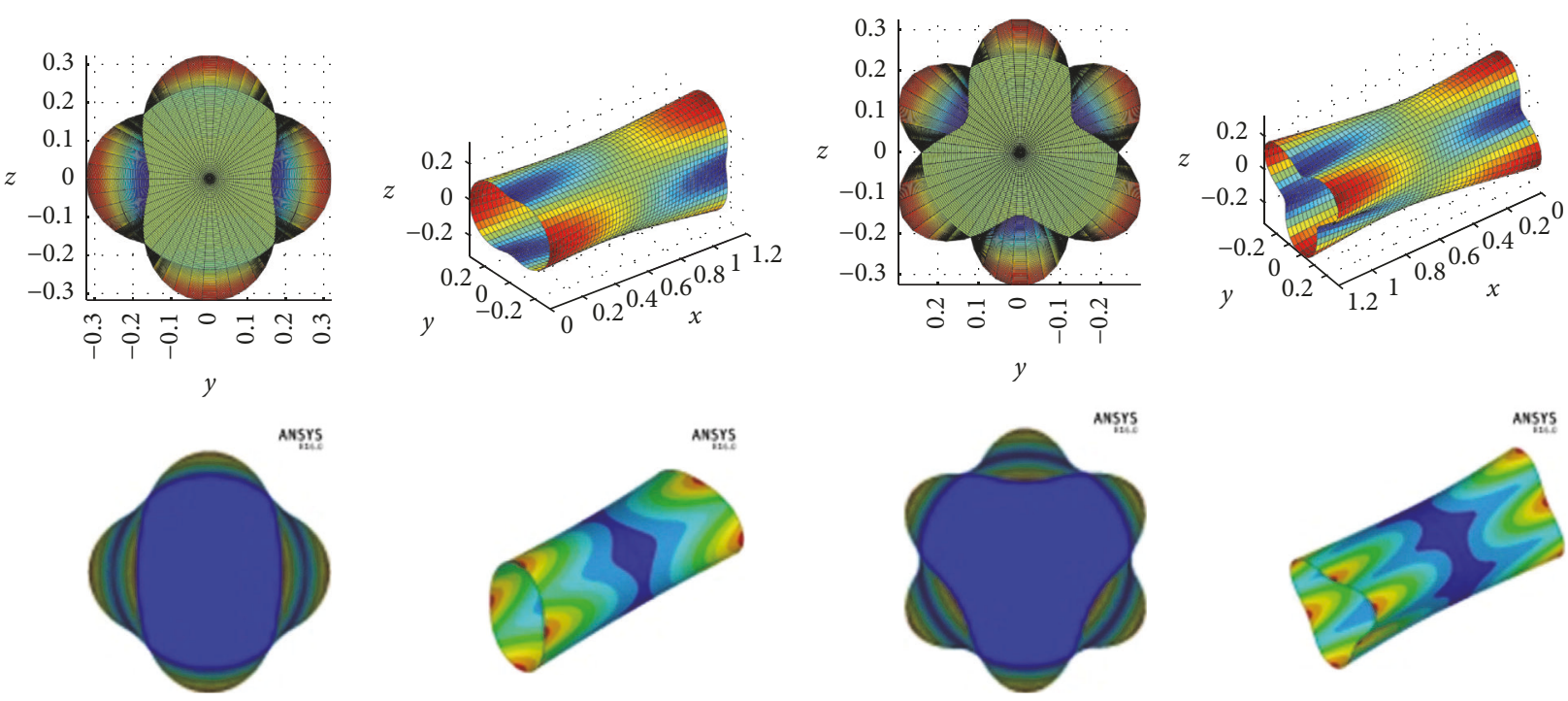

(a) $S(1,2)$

(b) $S(1,3)$
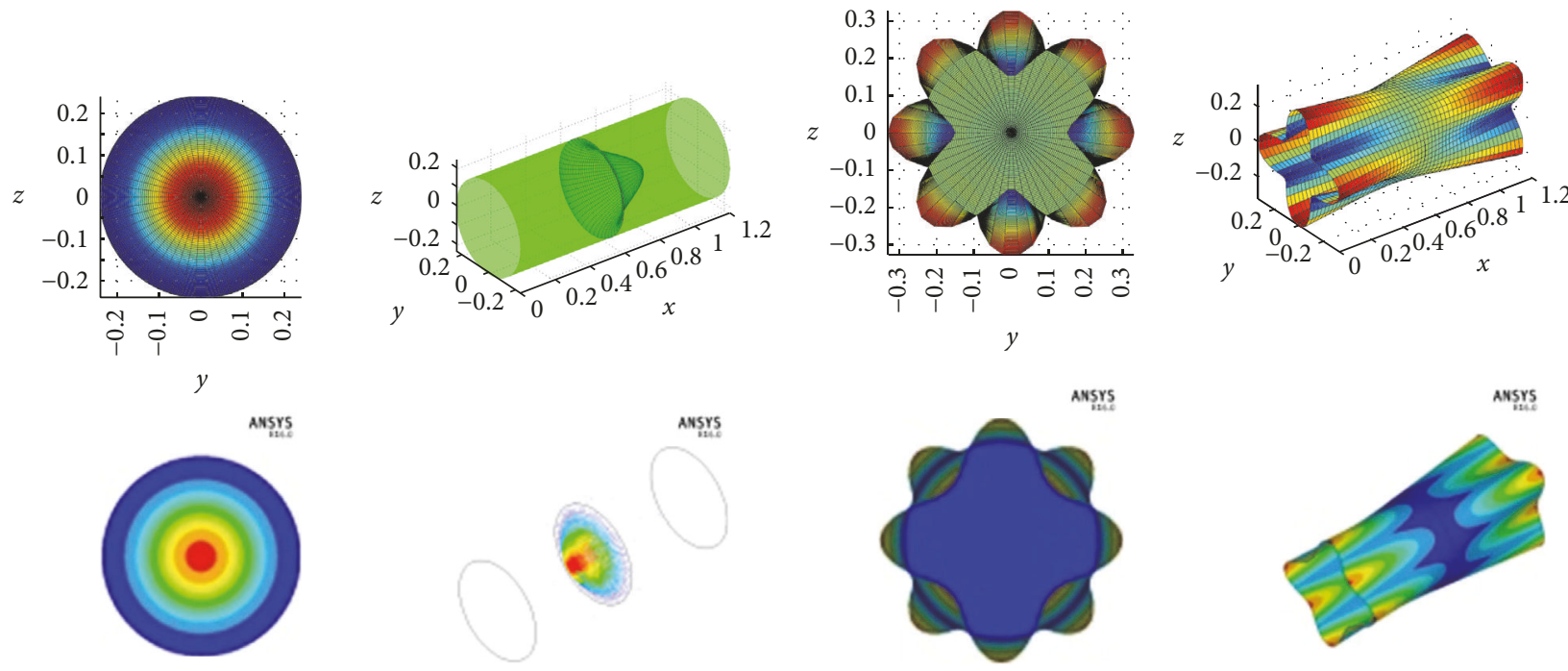

(c) $P(1,0)$

(d) $S(1,2)$
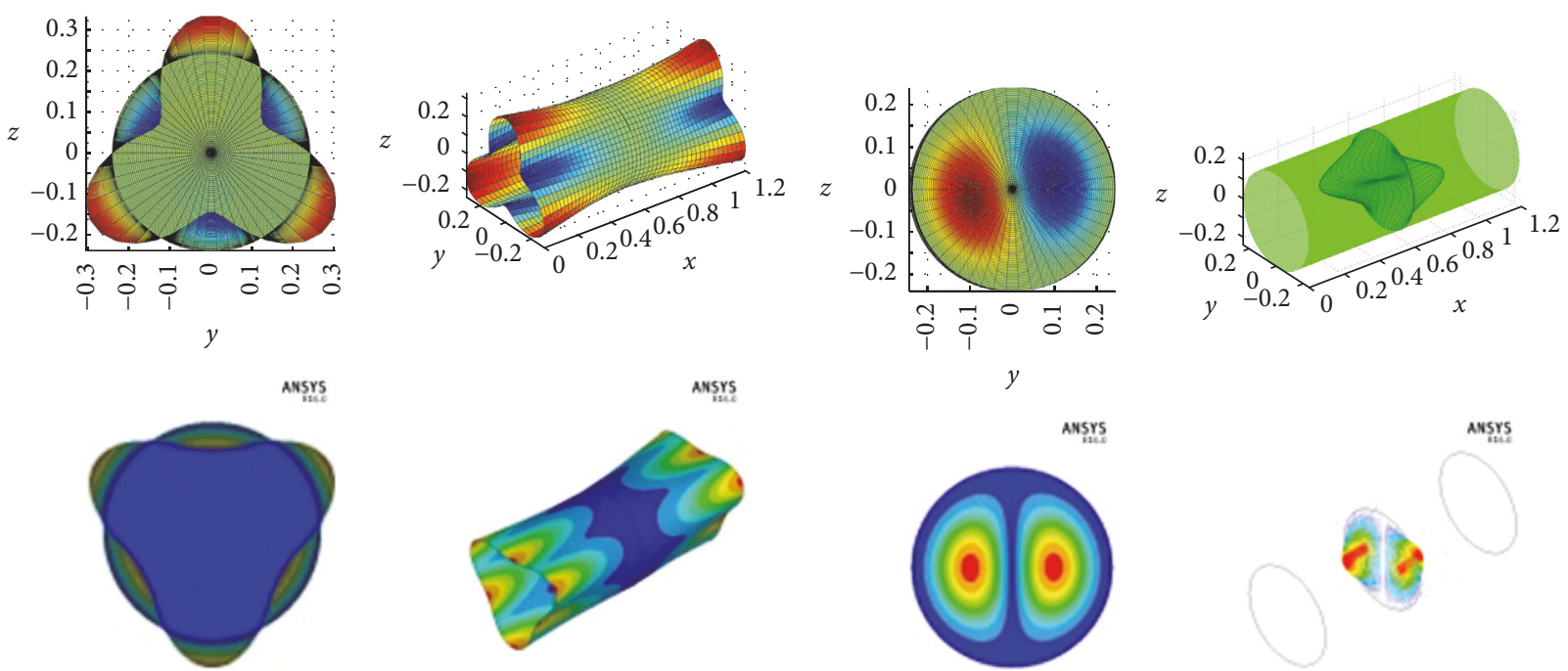

(e) $S(0,3)$

(f) $P(1,1)$

Figure 2: The modes of coupled structures with F-F. 

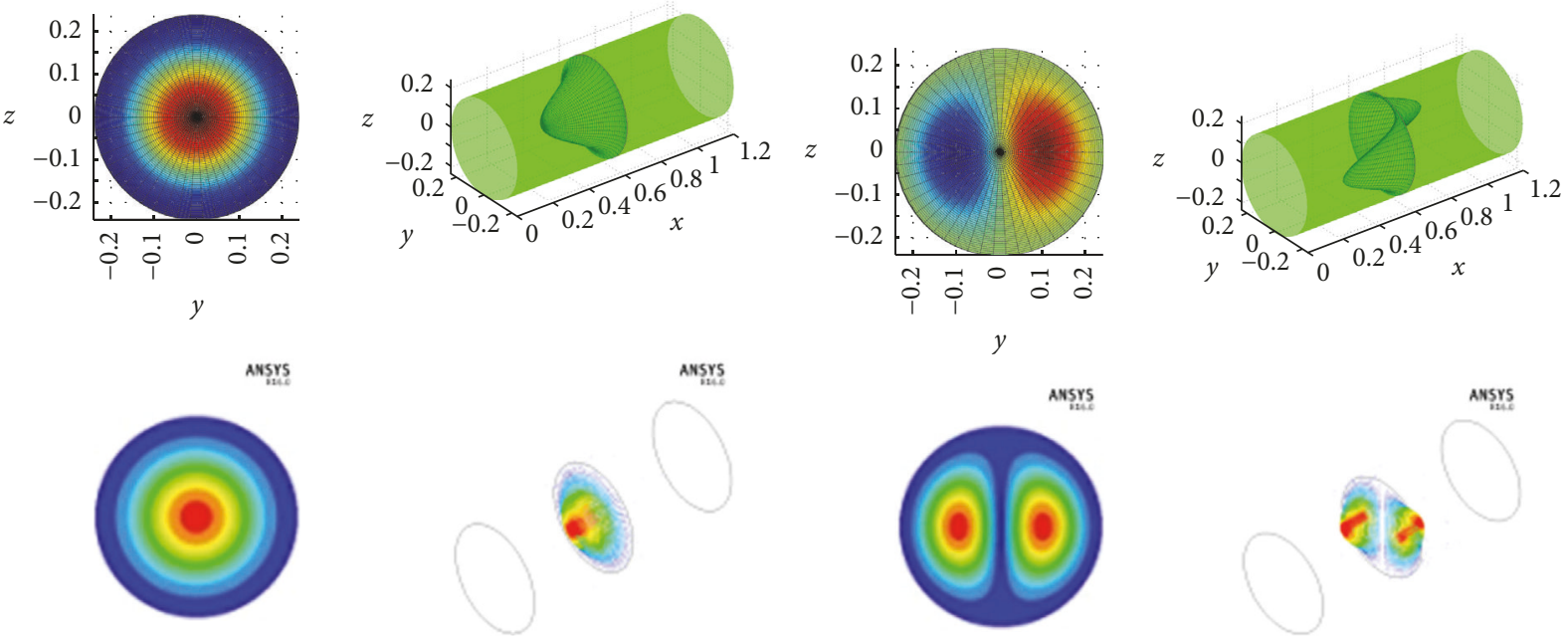

(a) $P(1,0)$

(b) $P(1,1)$
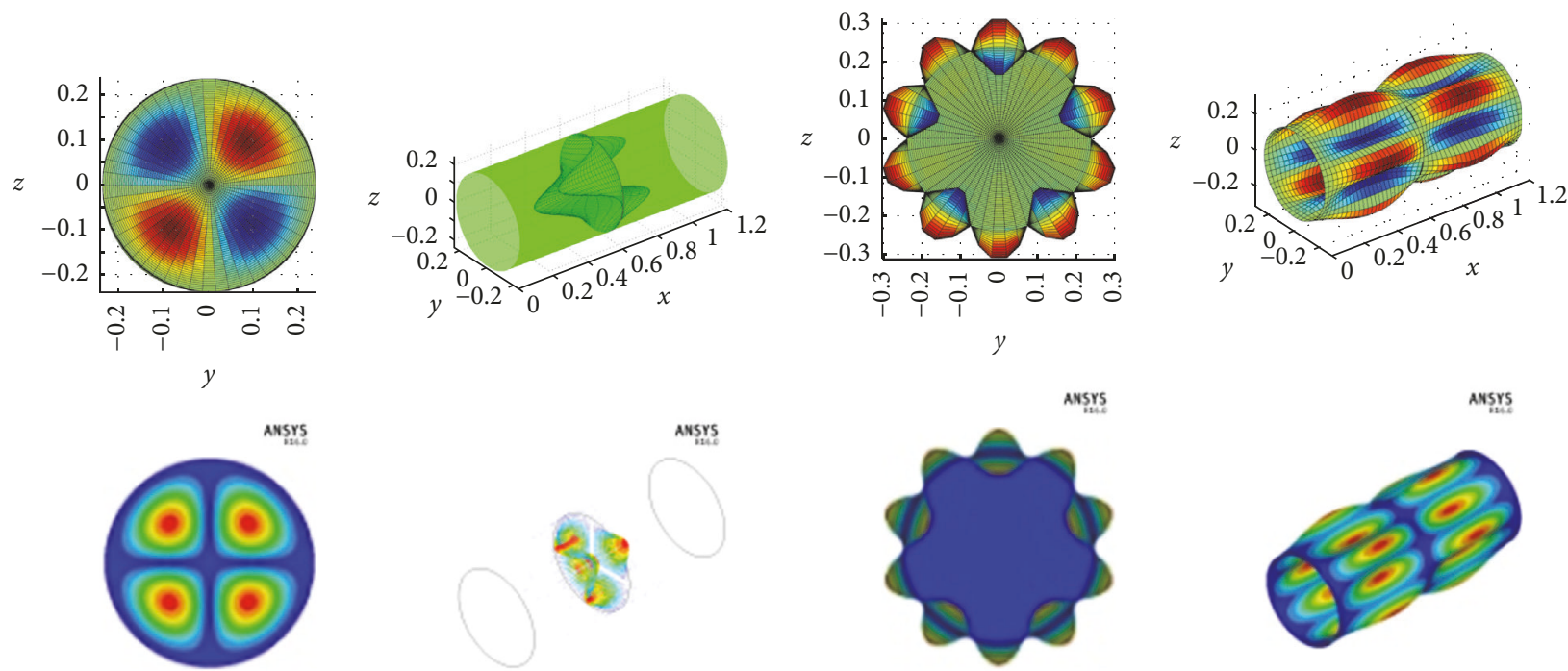

(c) $P(1,2)$
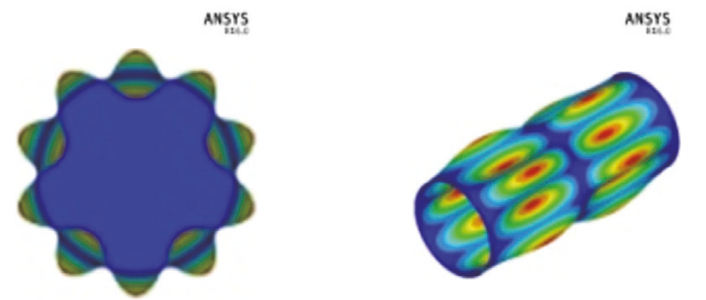

(d) $S(2,5)$
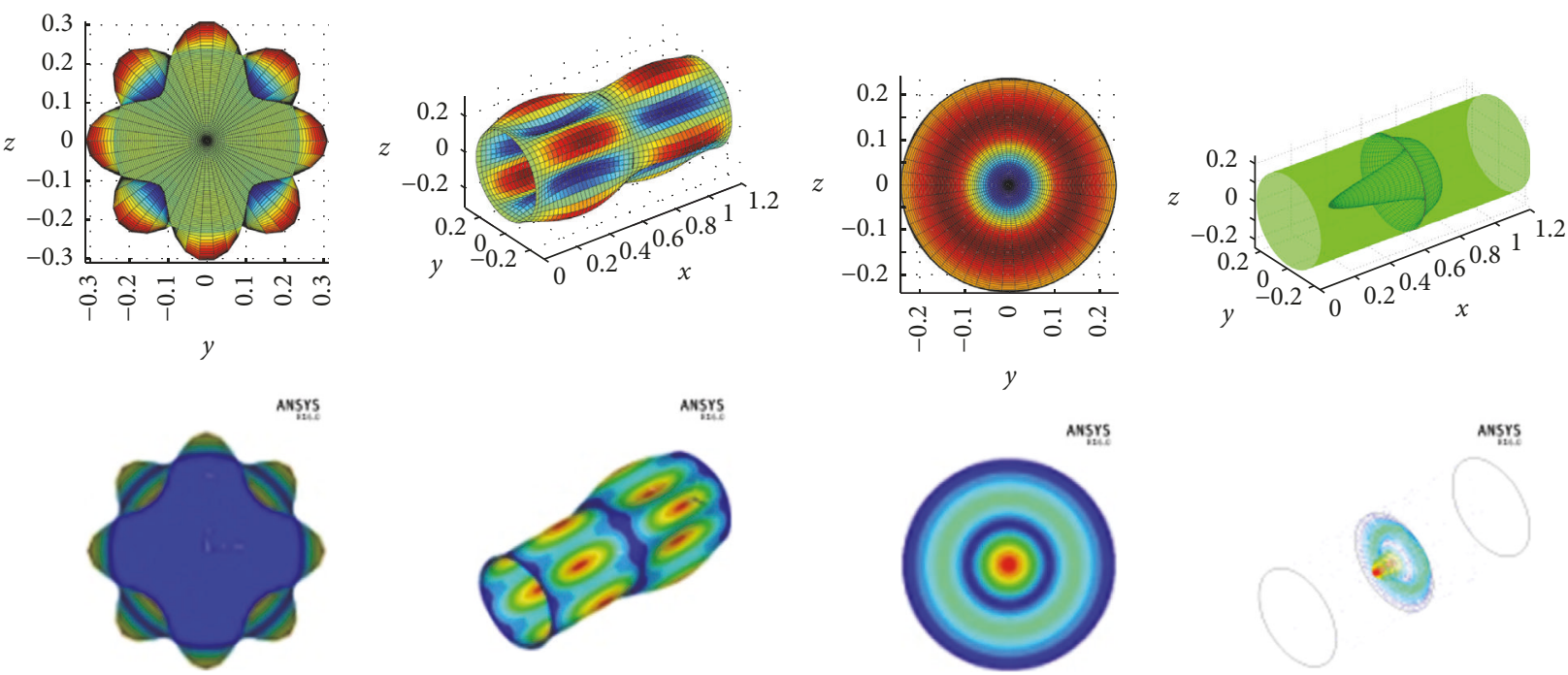

(e) $S(2,4)$

(f) $P(2,0)$

FIGURE 3: The modes of coupled structures with S-S. 

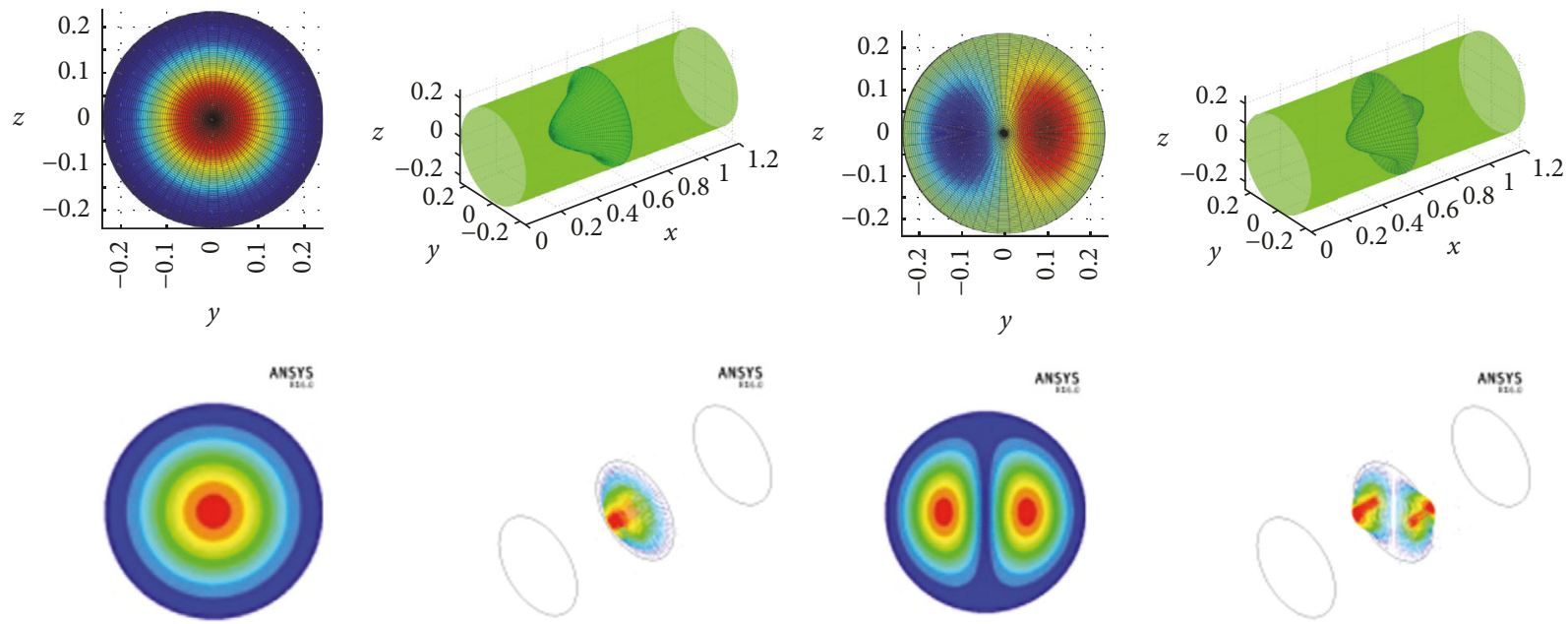

(a) $P(1,0)$

(b) $P(1,1)$
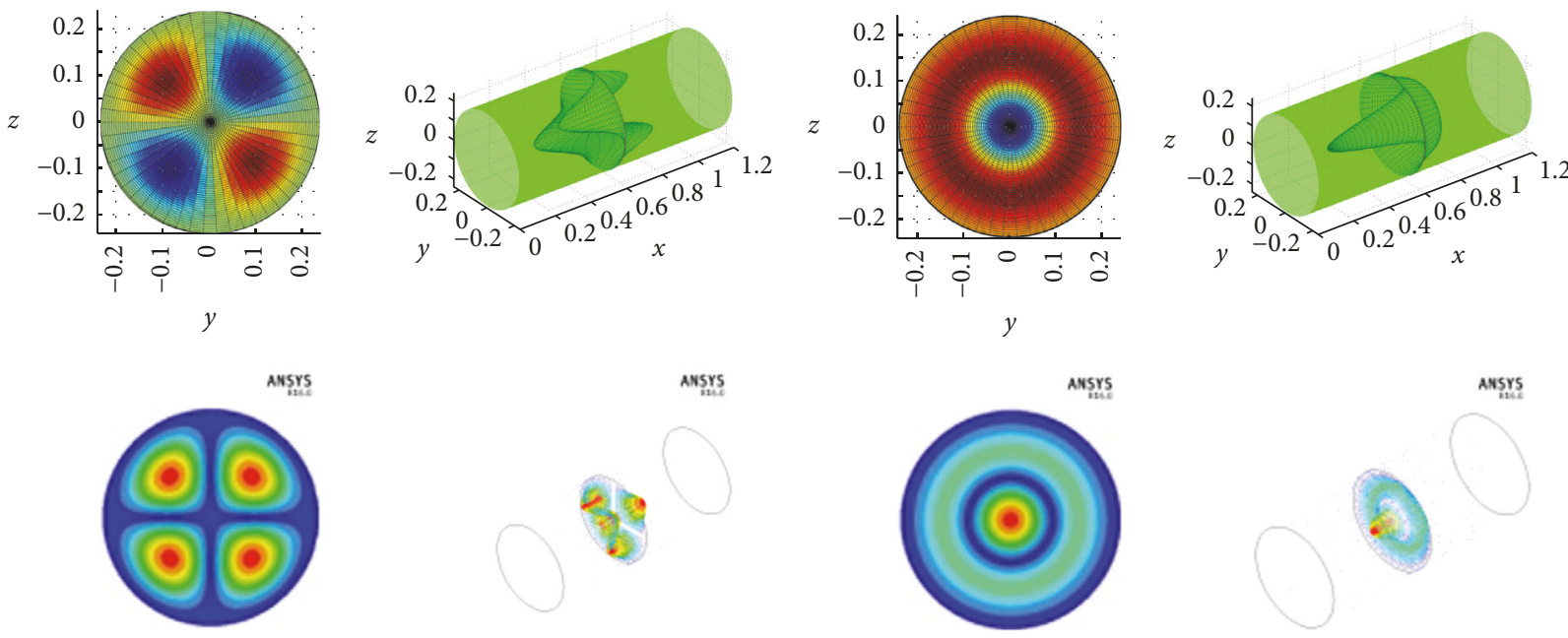

(c) $P(1,2)$

(d) $P(2,0)$
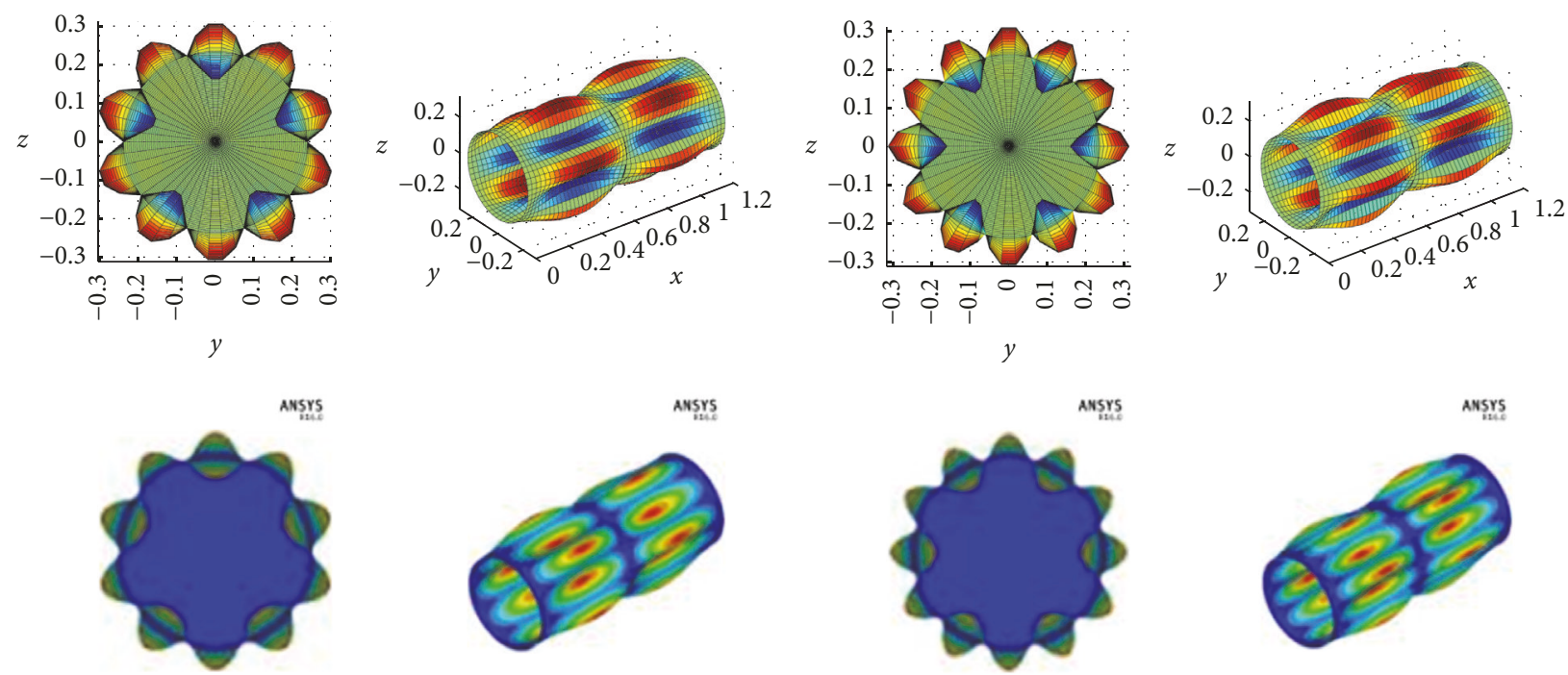

(e) $S(2,5)$

(f) $S(2,6)$

Figure 4: The modes of coupled structures with C-C. 

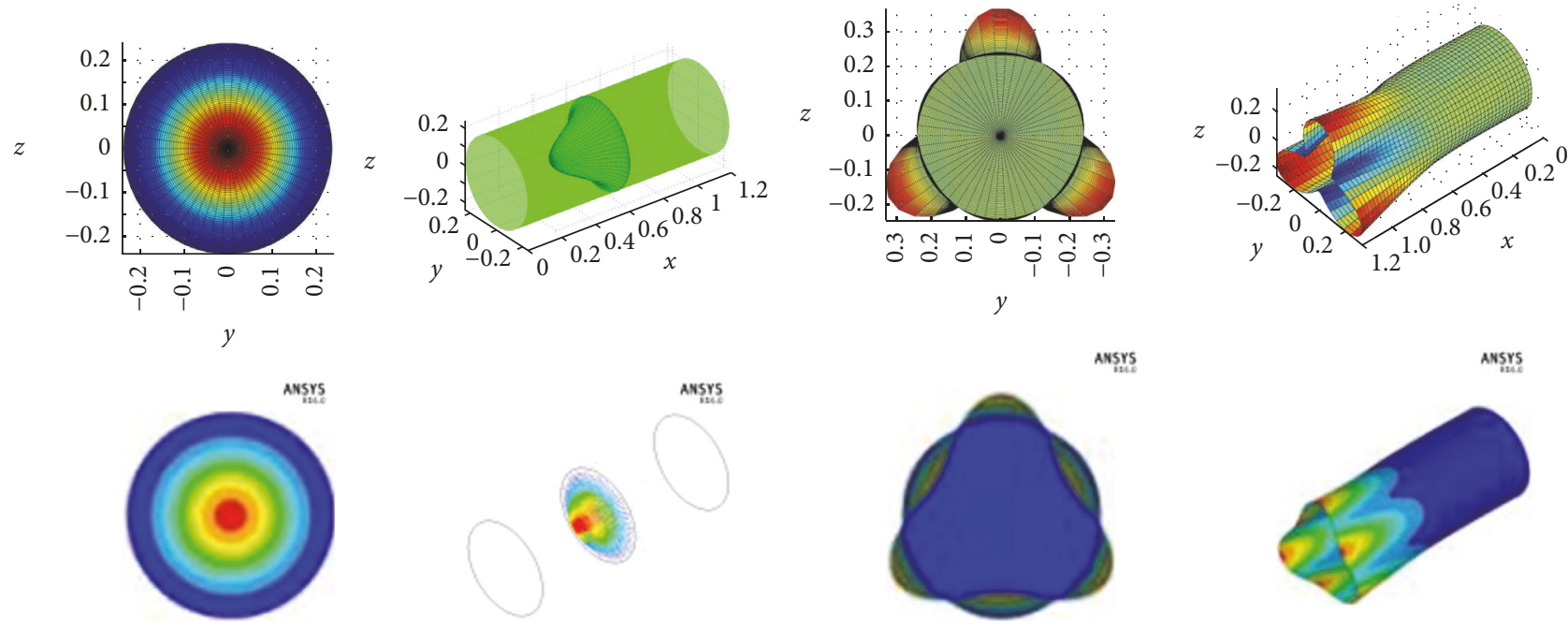

(a) $P(1,0)$

(b) $S(1,3)$
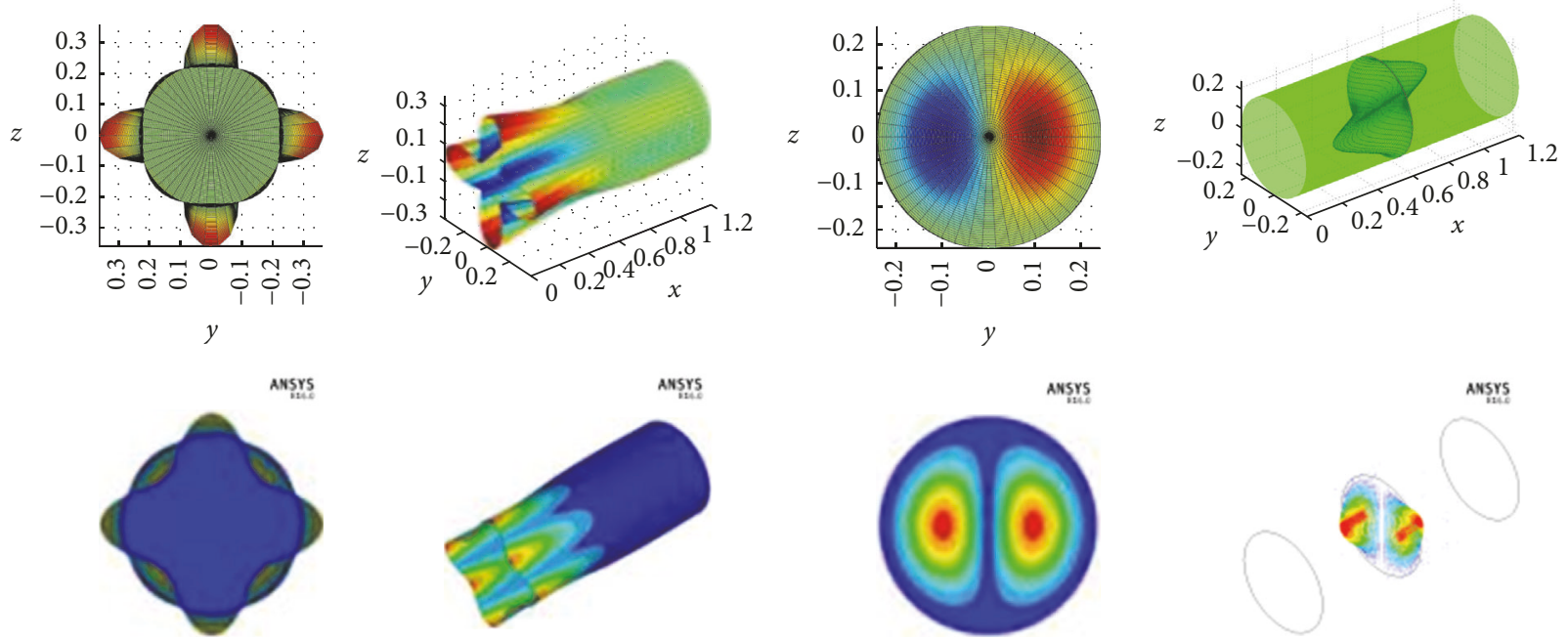

(c) $S(1,4)$

(d) $P(2,0)$
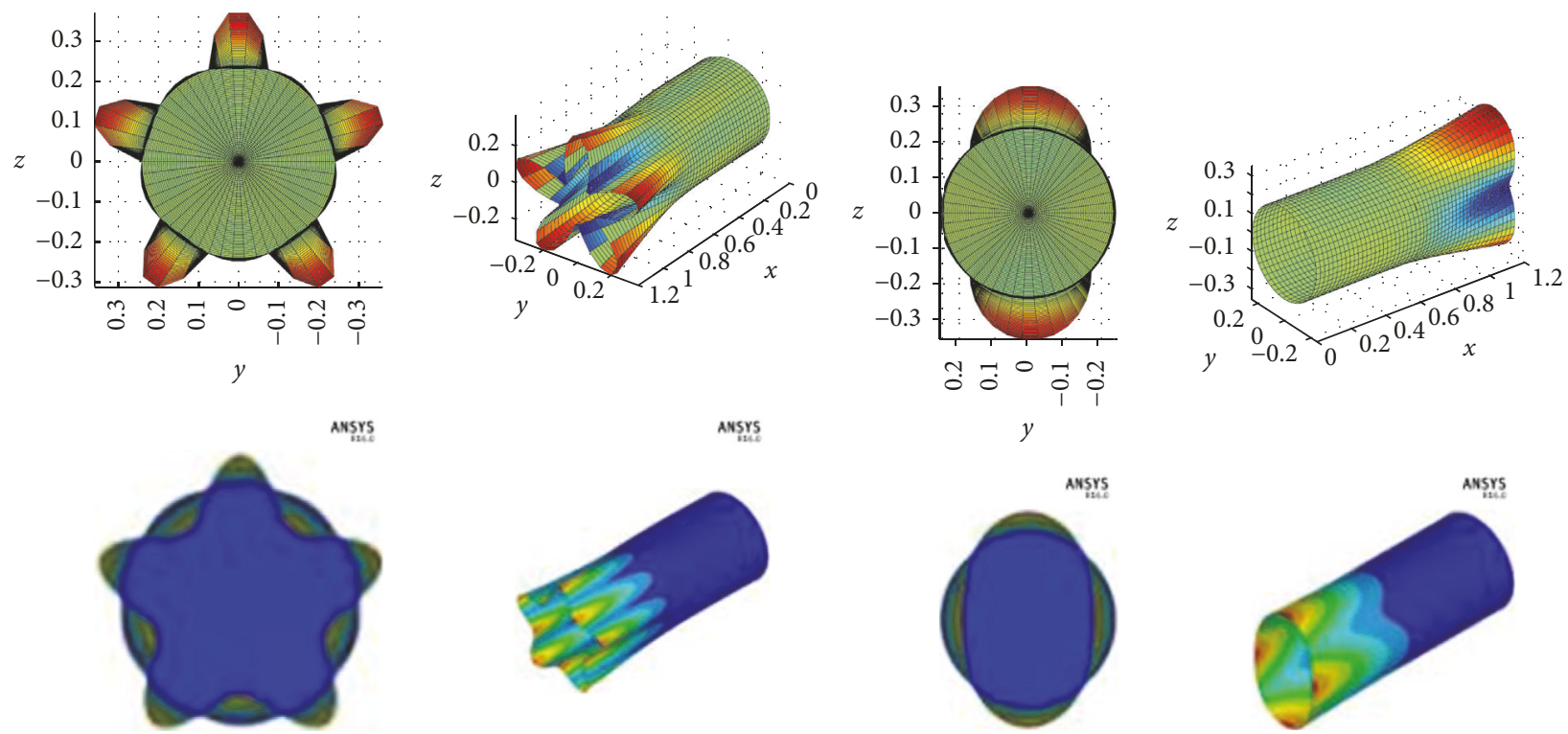

(e) $S(1,5)$
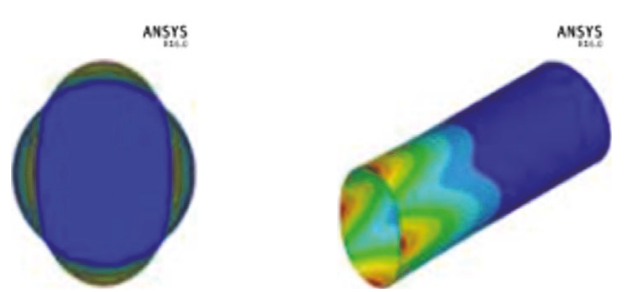

(f) $S(1,2)$

FIGURE 5: The modes of coupled structures with S-F. 

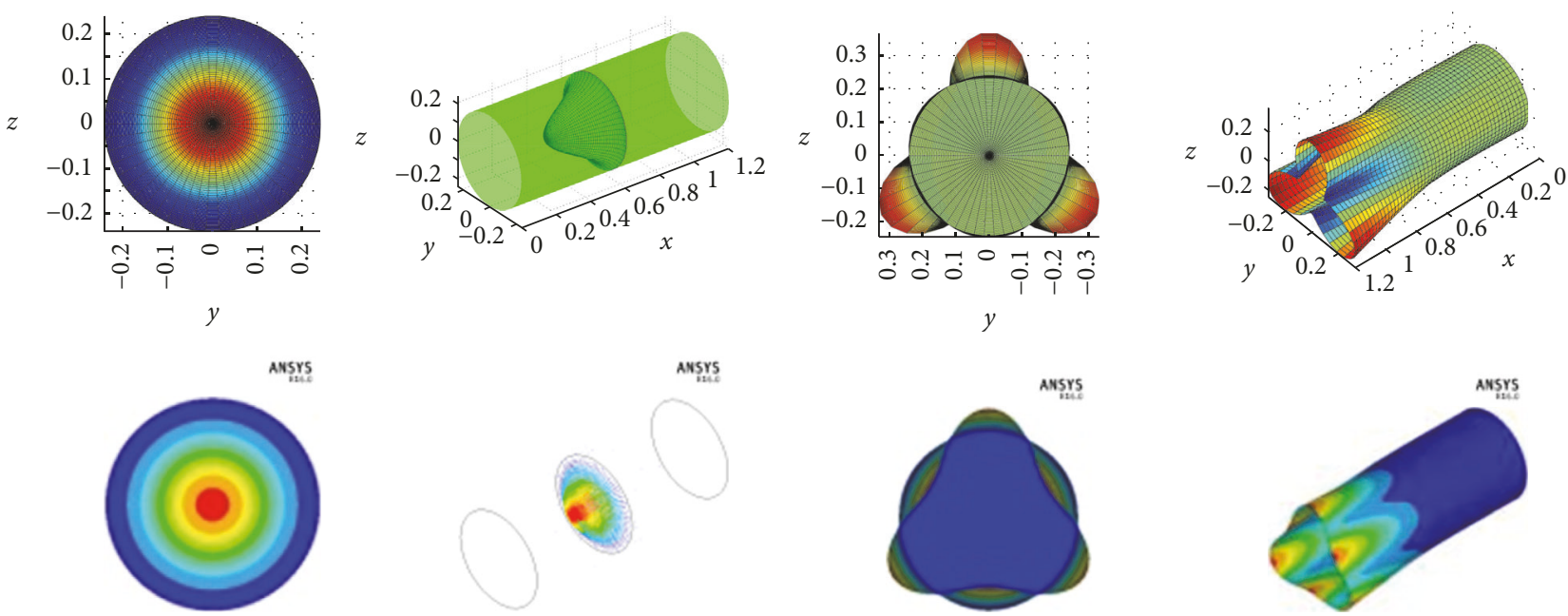

(a) $P(1,0)$

(b) $S(1,3)$
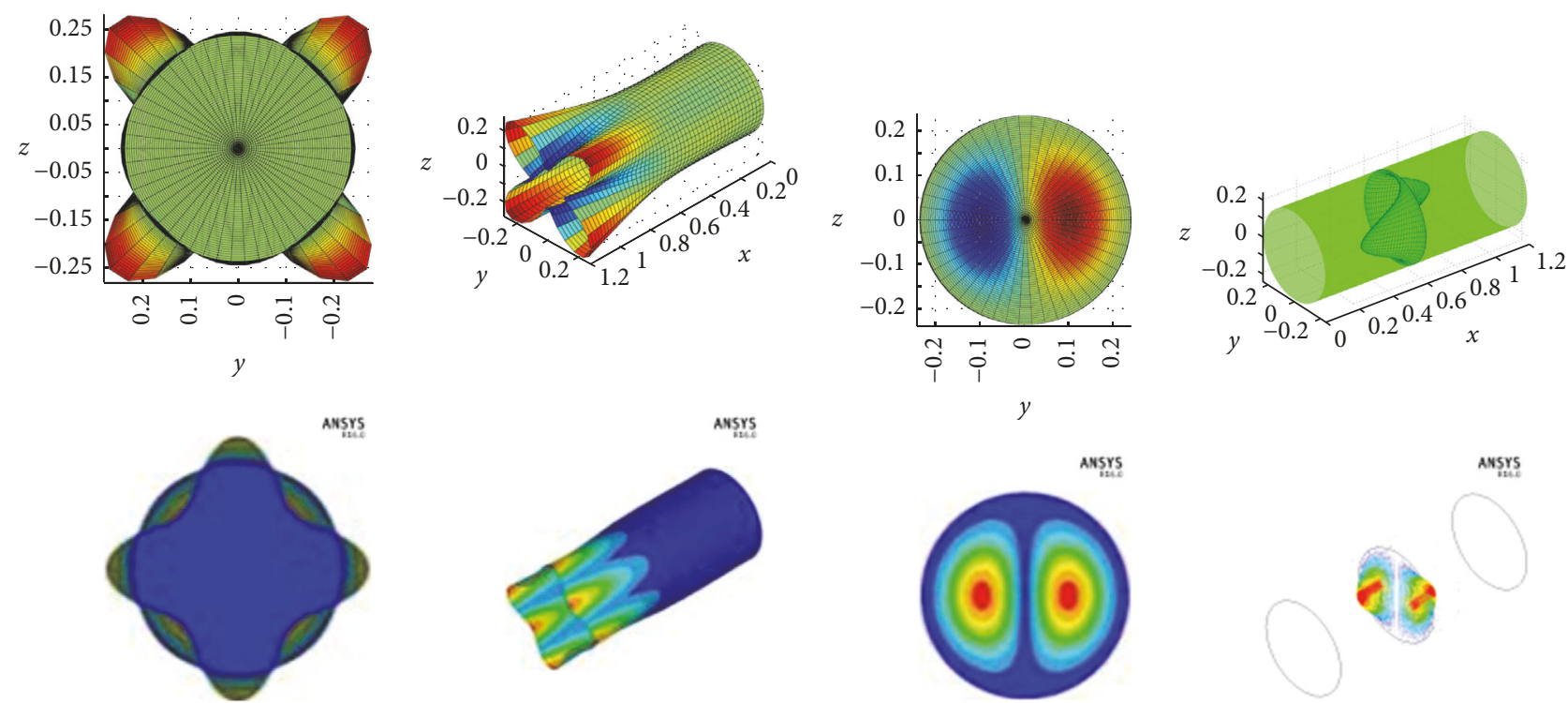

(c) $S(1,4)$
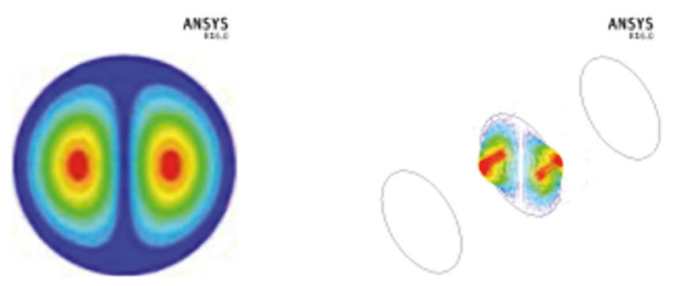

(d) $P(2,0)$
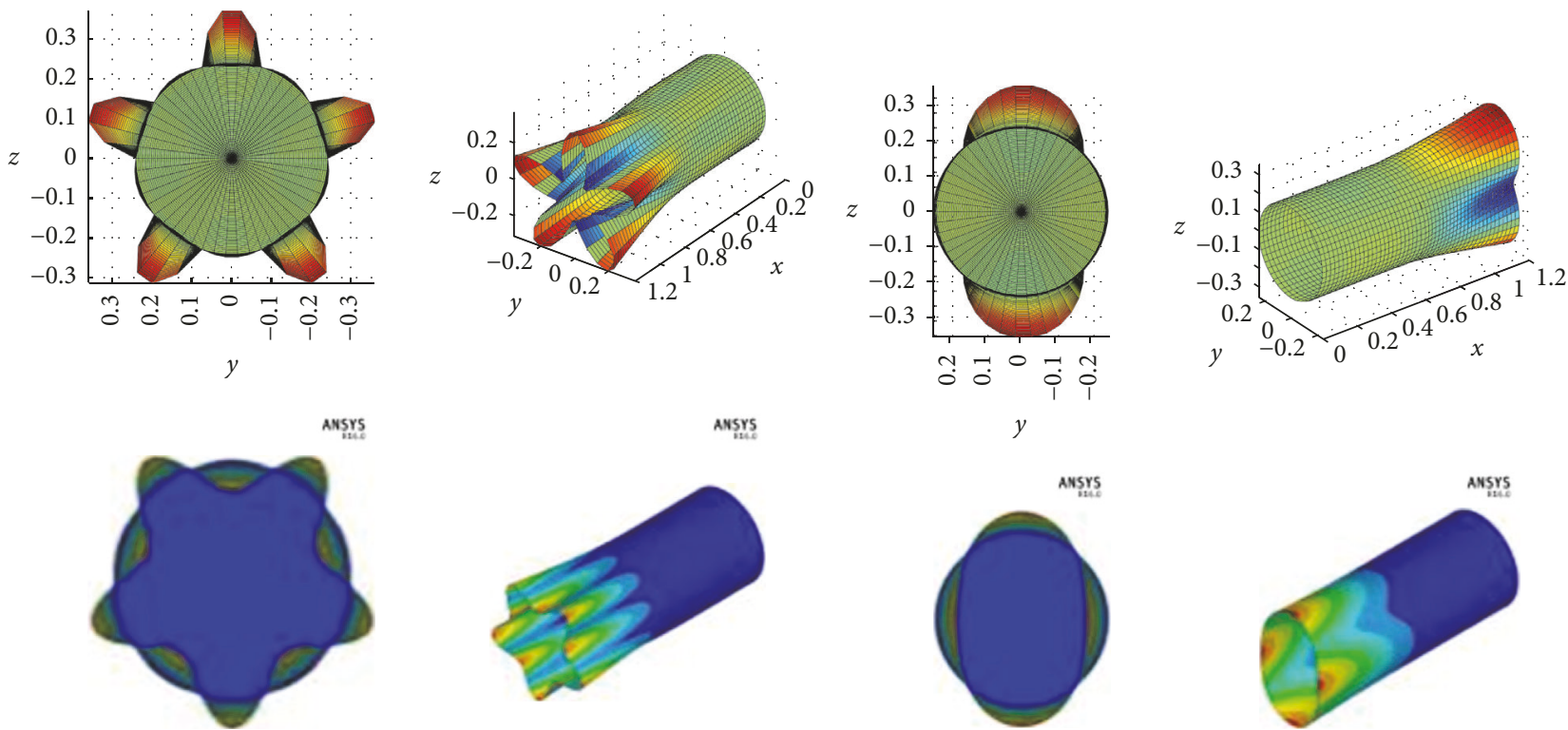

(e) $S(1,5)$

(f) $S(1,2)$

Figure 6: The modes of coupled structures with C-F. 

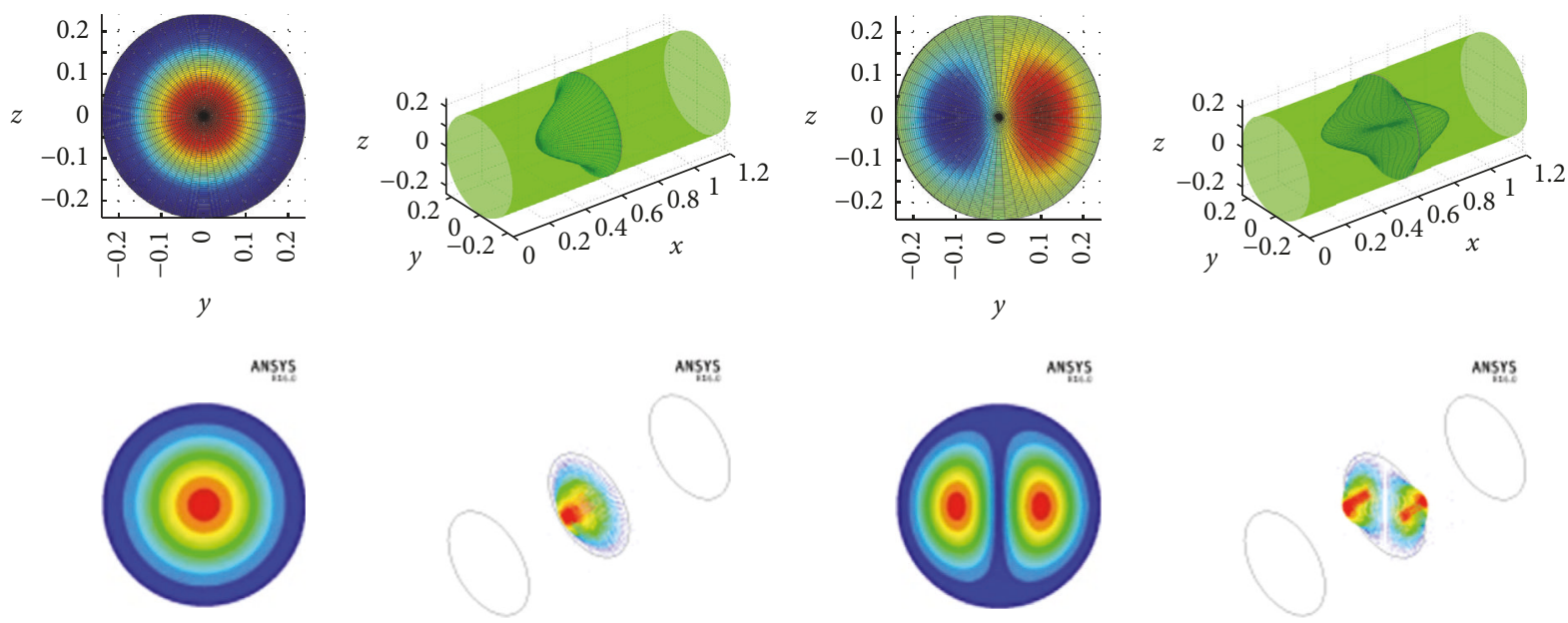

(a) $P(1,0)$

(b) $P(1,1)$
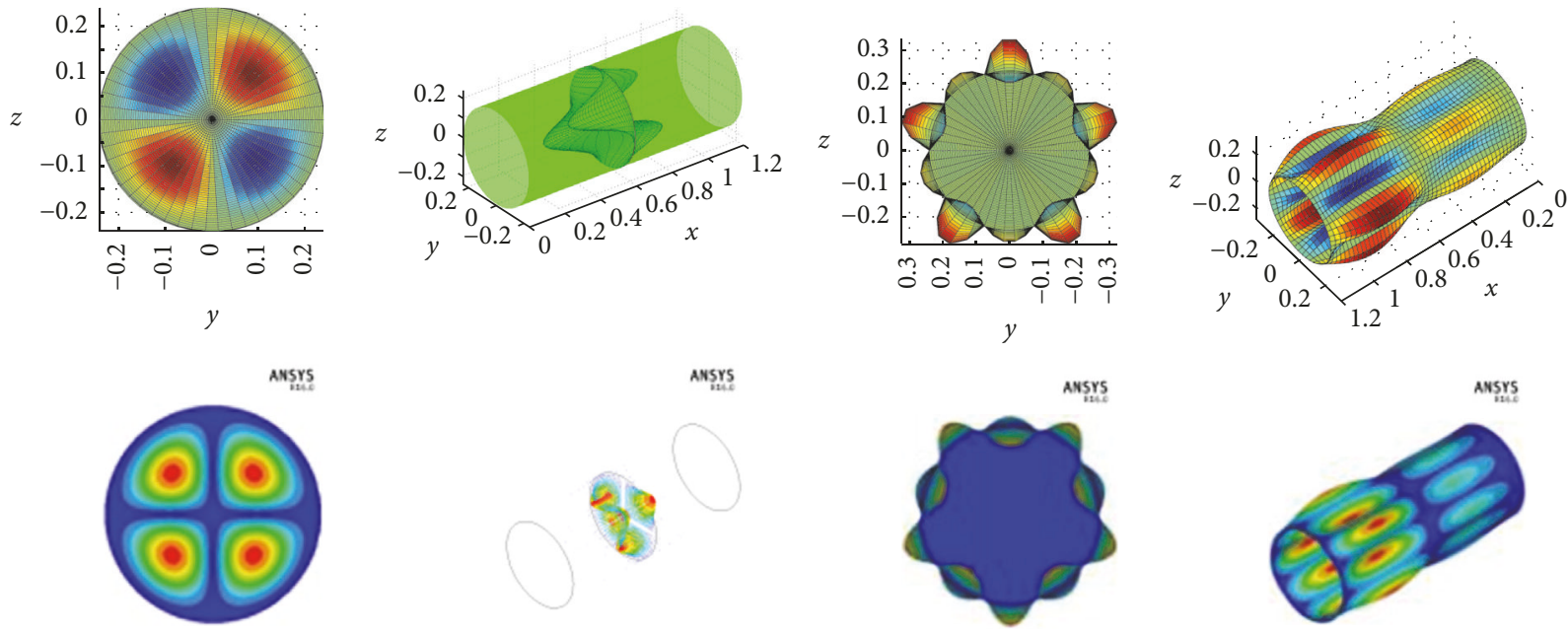

(c) $P(1,2)$

(d) $S(1,5)$
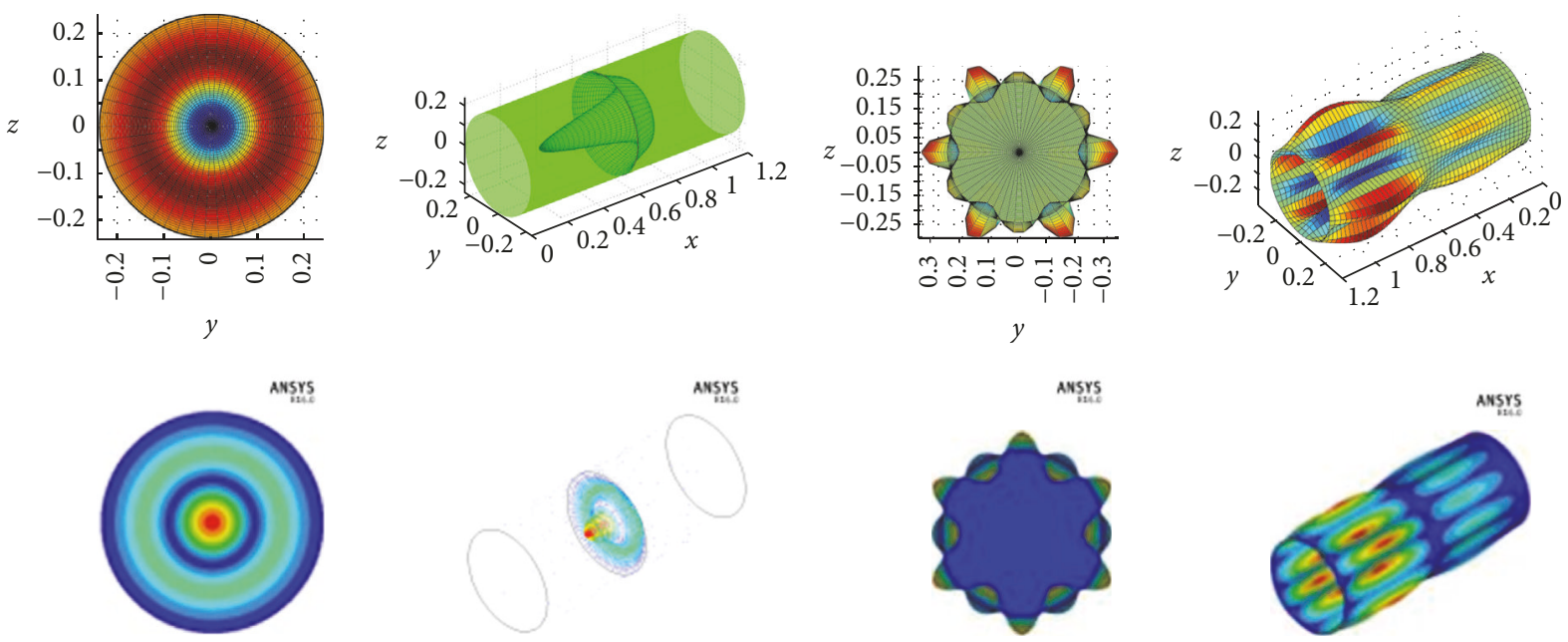

(e) $P(2,0)$

(f) $S(2,6)$

FIGURE 7: The modes of coupled structures with C-S. 


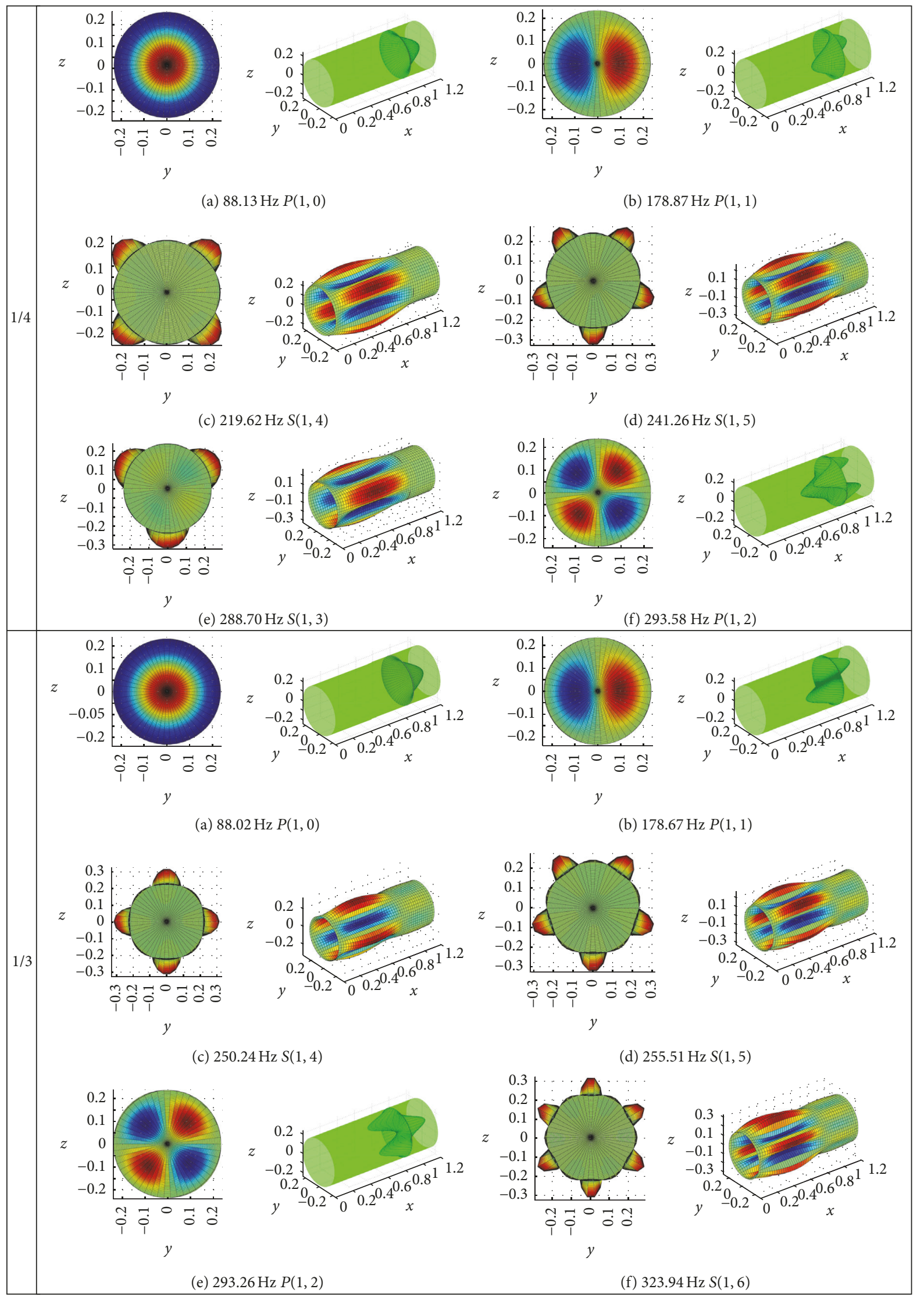

FIgUre 8: Continued. 


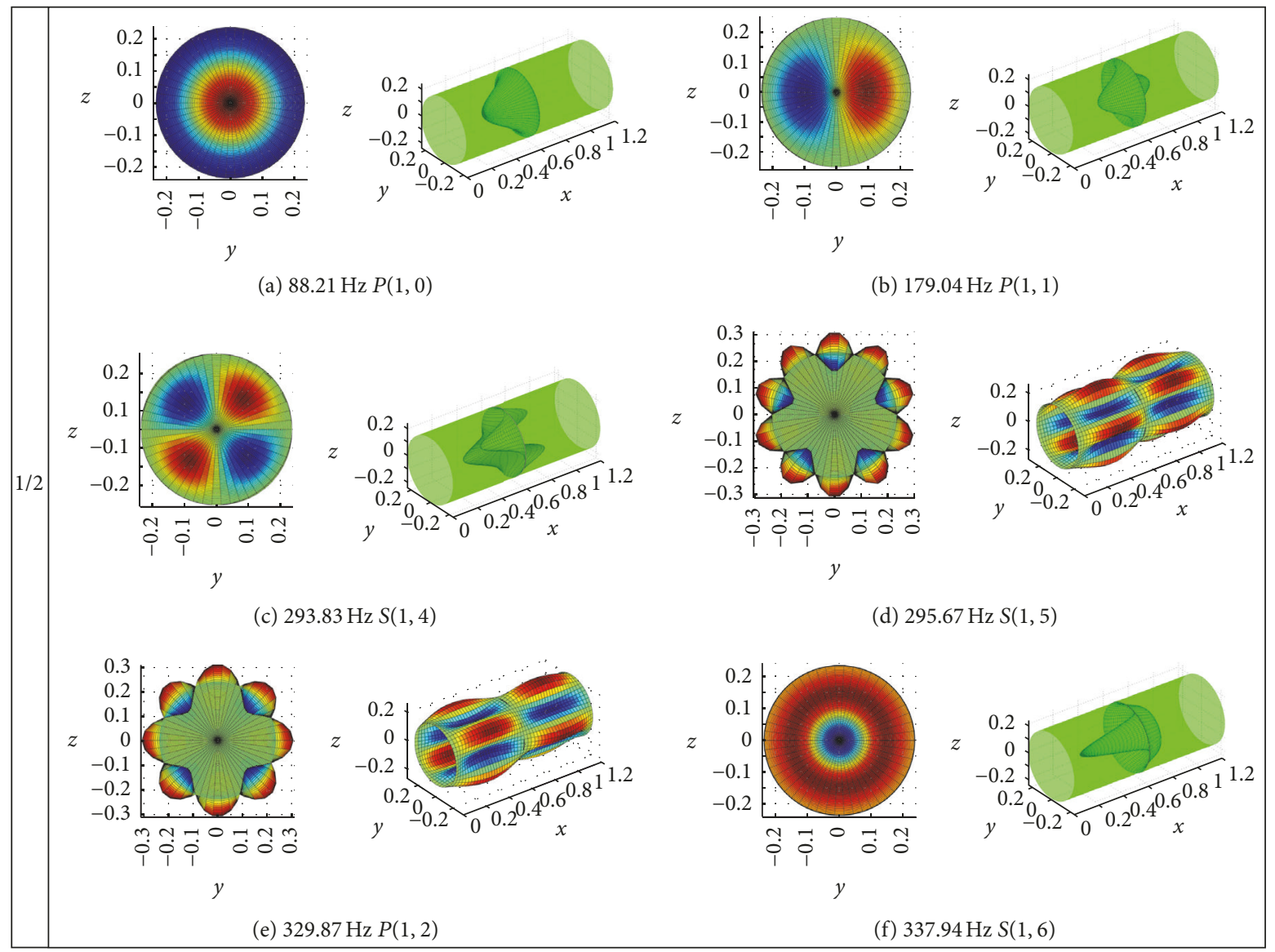

FIGURE 8: The modes of coupled structures with different coupling-junction positions.

effects of the boundary springs, we consider a cylindrical shell that is clamped at the right end and elastically supported at the left end and has only one style of spring attached in the corresponding direction. Clearly, the case in which the stiffness is zero represents clamp-free supported boundary conditions. As stated above, there is no correlation between the vibration characteristics of the circular plate and the boundary conditions of the coupled structure. Thus, the effects of the boundary spring that acts on the shell are the main consideration.

In Table 4, the first six natural frequencies are presented against the spring stiffness of the right end for all four support configurations. The frequencies are nearly equal when the stiffness of the rotational spring increases from 0 to $1 E 12$. The contribution of the rotational spring to the boundary restraint is relatively small. Relative to the rotational spring, the axial spring has a slight impact when the stiffness increases up to $1 E 10$. In comparison, the circumference and the radial spring have a larger influence on the boundary restraint. To further clarify the effects of the two types of spring on the boundary restraint, Figure 9 gives the influence of the two springs on the foundation frequency.

Table 4 shows that the first six natural frequencies increase continuously when the single spring stiffness changes from 0 to $1 E 12$. The natural frequencies are less

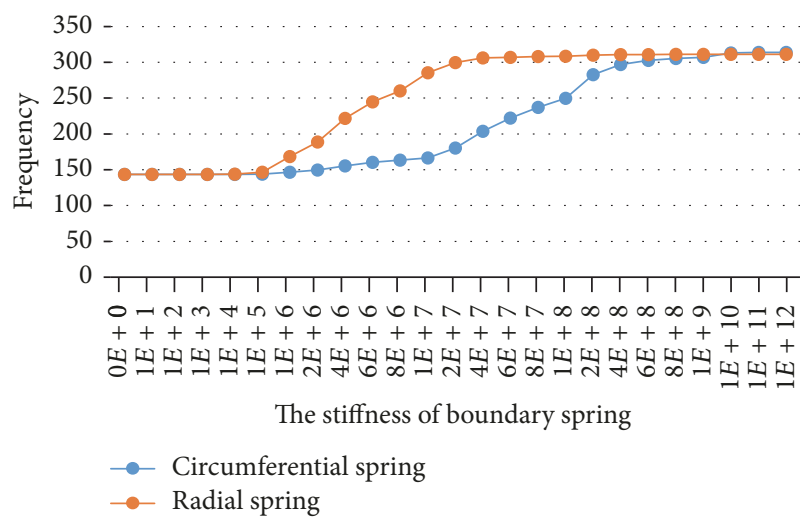

FIGURE 9: Natural frequency as a function of the stiffness of the radial and the circumferential linear springs.

sensitive to changes in the stiffness of the axial linear springs and the rotational springs. Relative to the axial linear springs and the rotational springs, the effects of the radial and the circumferential linear springs are larger for the natural frequencies of the coupling structure. To illustrate the effect of the sensitive springs, Figure 9 shows the natural frequencies of the coupled structure with the change of the stiffness of 
TABLE 4: Natural frequencies for a clamped, elastically supported shell $f / \mathrm{Hz}$.

\begin{tabular}{|c|c|c|c|c|c|c|c|}
\hline \multicolumn{2}{|c|}{ Stiffness } & (1) & (2) & (3) & $(4)$ & (5) & (6) \\
\hline \multirow{6}{*}{$k x$} & 0 & 143.65 & 150.16 & 213.20 & 233.85 & 265.82 & 304.44 \\
\hline & $1 E 4$ & 143.65 & 150.16 & 213.20 & 233.85 & 265.82 & 304.44 \\
\hline & $1 E 6$ & 143.71 & 150.18 & 213.21 & 233.99 & 265.98 & 304.44 \\
\hline & $1 E 8$ & 149.55 & 152.27 & 213.85 & 246.05 & 279.07 & 304.66 \\
\hline & $1 E 10$ & 176.32 & 205.89 & 222.30 & 307.96 & 340.41 & 345.43 \\
\hline & $1 E 12$ & 179.66 & 212.57 & 223.64 & 308.56 & 343.98 & 355.50 \\
\hline \multirow{6}{*}{$k \theta$} & 0 & 143.65 & 150.16 & 213.20 & 233.85 & 265.82 & 304.44 \\
\hline & $1 E 4$ & 143.68 & 150.18 & 213.21 & 233.89 & 265.86 & 304.44 \\
\hline & $1 E 6$ & 146.66 & 151.91 & 214.02 & 237.27 & 269.57 & 304.84 \\
\hline & $1 E 8$ & 249.71 & 263.48 & 303.81 & 329.33 & 385.81 & 394.80 \\
\hline & $1 E 10$ & 313.07 & 352.82 & 357.63 & 406.39 & 406.66 & 438.04 \\
\hline & $1 E 12$ & 313.74 & 353.13 & 359.17 & 406.56 & 406.96 & 438.04 \\
\hline \multirow{6}{*}{$k r$} & 0 & 143.65 & 150.16 & 213.20 & 233.85 & 265.82 & 304.44 \\
\hline & $1 E 4$ & 143.93 & 150.45 & 213.41 & 233.99 & 265.86 & 304.58 \\
\hline & $1 E 6$ & 168.50 & 175.18 & 231.25 & 247.30 & 269.57 & 316.69 \\
\hline & $1 E 8$ & 308.64 & 338.51 & 351.82 & 386.27 & 404.76 & 405.85 \\
\hline & $1 E 10$ & 311.26 & 348.03 & 352.67 & 405.93 & 406.30 & 437.91 \\
\hline & $1 E 12$ & 311.29 & 348.12 & 352.68 & 405.94 & 406.30 & 437.91 \\
\hline \multirow{6}{*}{$K r$} & 0 & 143.65 & 150.16 & 213.20 & 233.85 & 265.82 & 304.44 \\
\hline & $1 E 4$ & 144.59 & 151.31 & 214.23 & 234.31 & 265.84 & 305.34 \\
\hline & $1 E 6$ & 146.31 & 153.31 & 215.90 & 235.17 & 265.87 & 306.67 \\
\hline & $1 E 8$ & 146.36 & 153.37 & 215.94 & 235.20 & 265.88 & 306.70 \\
\hline & $1 E 10$ & 146.36 & 153.37 & 215.94 & 235.20 & 265.87 & 306.70 \\
\hline & $1 E 12$ & 146.36 & 153.37 & 215.94 & 235.20 & 265.88 & 306.70 \\
\hline
\end{tabular}

the radial and the circumferential linear springs. When the stiffness of the spring changes from 0 to $1 E 6$, the stiffness is so small that there is no difference between the natural frequencies of the C-E boundary condition and those of the $\mathrm{C}-\mathrm{F}$ boundary condition. When the stiffness of the spring is higher than $1 E 10$, the stiffness is so large that the elastic boundary condition can be viewed as rigid. The curves in Figure 9 are relatively steep when $1 E 6<K<1 E 10$ because the natural frequencies are much more sensitive to the change of the stiffness in this range for the current set of parameters.

The $S(1,5)$ mode shapes for the radial spring stiffness and circumferential spring stiffness are plotted in Figures 10 and 11 , respectively. The mode shapes are significantly modified by the stiffness of the restraining springs. When the boundary condition changes from C-E (with a circumferential spring stiffness of 1E4) to $\mathrm{C}-\mathrm{C}$, the natural frequency changes accordingly from approximately $213 \mathrm{~Hz}$ to approximately $358 \mathrm{~Hz}$. The same phenomenon can be observed in Figure 11.

3.4. Forced Vibration Analyses. Three points, Point A $(0.24,0$, $0.2)$ and Point $B(0.24,0,0.9)$ in the cylindrical shell and Point $\mathrm{C}(0.12,0,0.6)$ in the middle circular plate, are introduced in the respective local coordinate systems. A unit harmonic force $f s=e^{j \omega t}$ is applied on the cylindrical shell at Point A. The accuracy of the proposed model is validated through comparison with the finite-element program ANSYS. The finite-element model of the cylindrical shell-plate coupled structure, which consists of 4-node SHELL181 elements, is meshed into 15360 elements to obtain reasonably convergent results. The full calculation procedure (direct solver) is implemented in ANSYS.

Figures 12 and 13 show the comparisons of responses at Points $\mathrm{B}$ and $\mathrm{C}$ for the coupled structure subjected to the normal point forces at Point A between ANSYS and the proposed method. The responses of the cylindrical shellplate coupled structure subjected to a point force show many resonant peaks. This behavior is expected since these external loads can excite both symmetric and antisymmetric vibration modes of the coupled structure. Since no damping has been introduced in the proposed and finite-element models, the resonant peaks should in theory reach an infinite level but are limited due to the chosen frequency-calculation step. Therefore, the amplitudes of the resonant peaks are not significant because they are strongly affected by the finite discretization of the frequency range. Except for the locations of the resonant peaks, the displacement responses of the two methods are in excellent agreement, which validates the accuracy of the proposed method in predicting the forced vibration of the coupled structure. It should be noted that the computational time and the number of degrees of freedom (DOFs) accounts for the advantages of the proposed method over the traditional FEM. In the calculation, only 2772 DOFs are needed for the proposed method, while ANSYS requires more than 90000 DOFs. Thus, the proposed computational procedure is simple and effective, which will make it of great interest to engineers. 


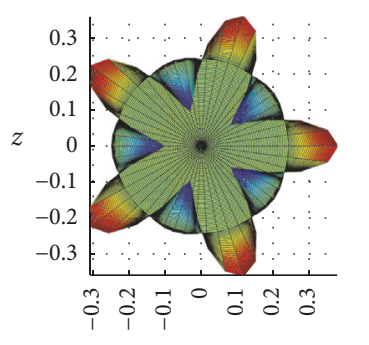

$y$

(a)
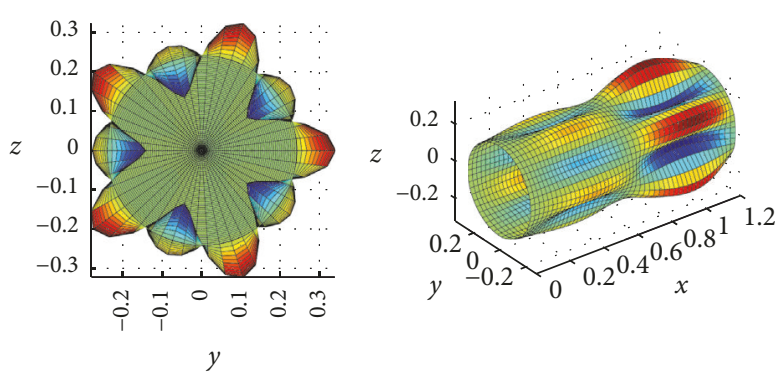

(c)
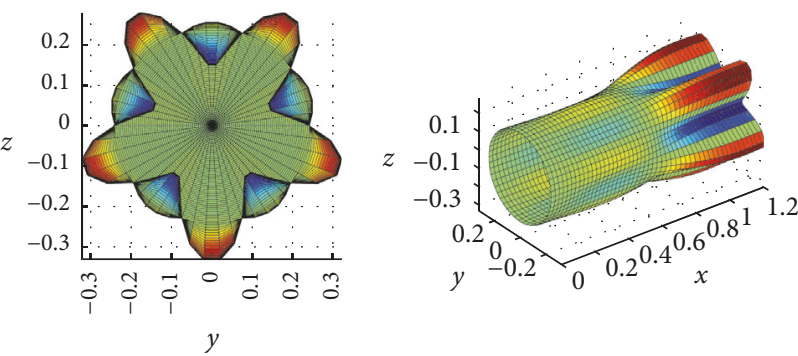

(b)
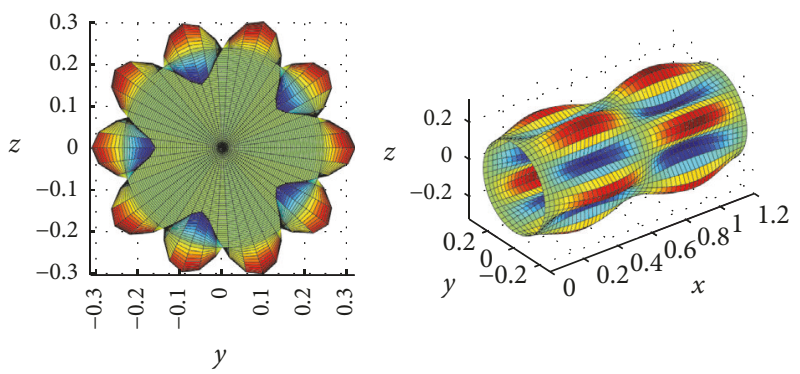

(d)

Figure 10: The $S(1,5)$ modes of coupled structures with C-E boundary condition: (a) $k x=k r=K r=0$ and $k \theta=1 E 4$, 213.21 Hz; (b) $k x=k r=K r=0$ and $k \theta=1 E 8,263.48 \mathrm{~Hz}$; (c) $k x=k r=K r=0$ and $k \theta=1 E 12,313.74 \mathrm{~Hz}$; and (d) C-C, $358.82 \mathrm{~Hz}$.

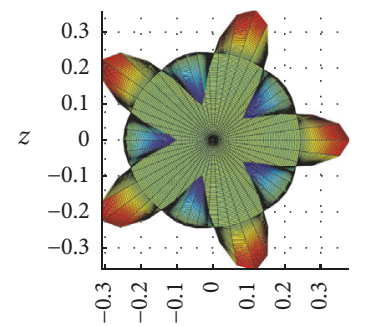

$y$

(a)

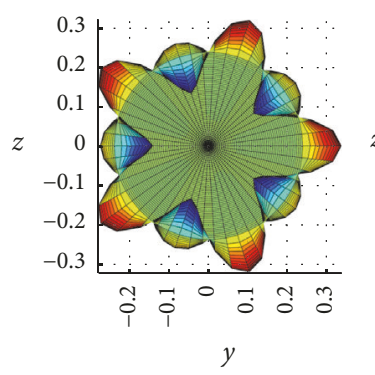

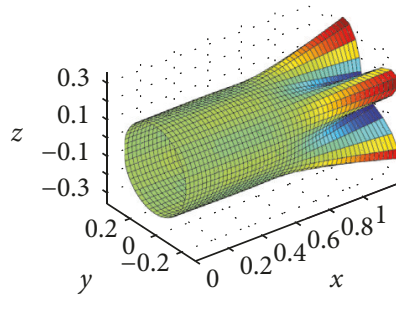

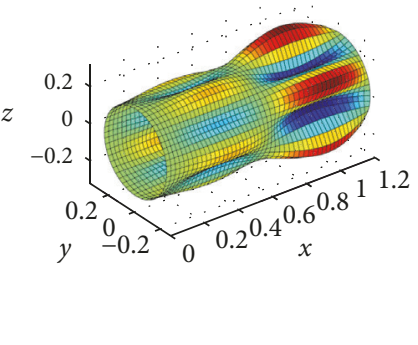

(c)
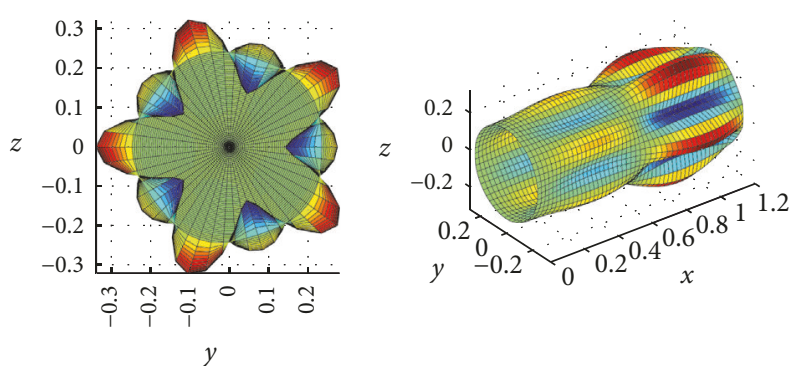

(b)
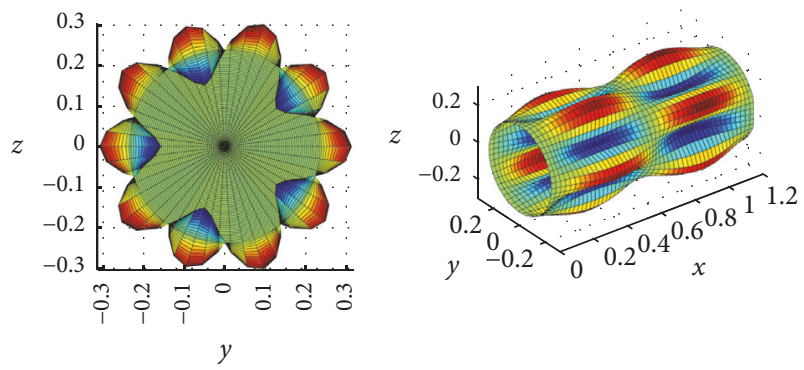

(d)

Figure 11: The $S(1,5)$ modes of coupled structures with C-E boundary condition: (a) $k x=k \theta=K r=0$ and $k r=1 E 4,213.41 \mathrm{~Hz}$; (b) $k x=k \theta=K r=0$ and $k r=1 E 8,308.64 \mathrm{~Hz}$; (c) $k x=k \theta=K r=0$ and $k r=1 E 12,311.27 \mathrm{~Hz}$; and (d) C-C, $358.82 \mathrm{~Hz}$.

As noted in Section 3.3, the stiffness of the boundary springs can have a significant impact on the modal characteristics of the coupled structure. The responses of the coupled structure for different stiffness values are shown in Figure 14. The figures indicate that the heights of the resonance peaks monotonically increase with the stiffness of the boundary springs. In a comparison of Figures 14(a) and 14(b), the increase of $k v 1$ is more easily detected near the stiffness $1 E 8$. The phenomenon can be explained by referring to Figure 9 .

Figure 15 shows the responses of the coupled structure for different values of coupling-spring stiffness. The stiffness of all four types of spring is increased from $1 E 7$ to $1 E 9$. The 


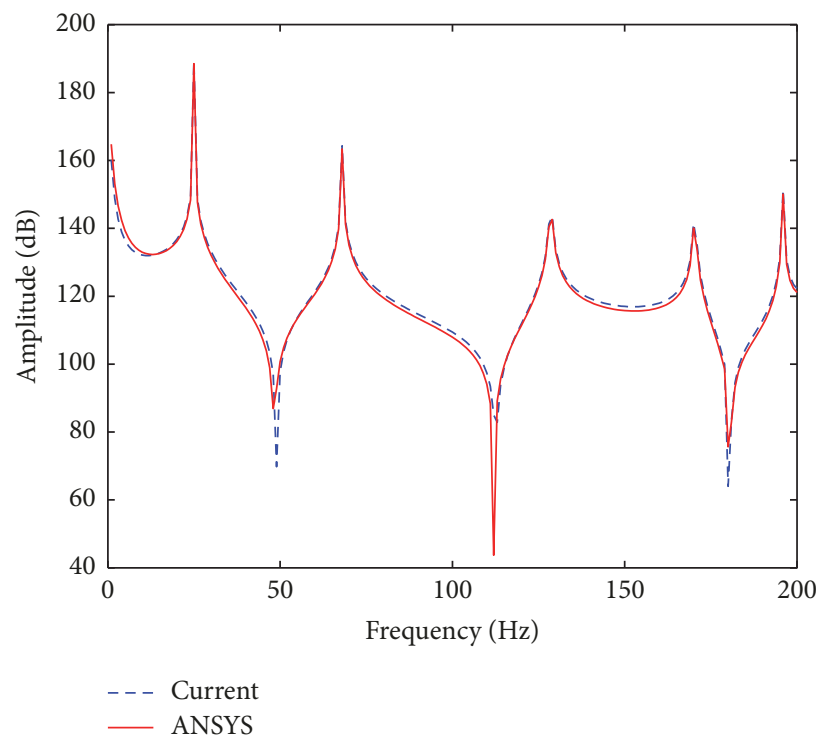

FIGURE 12: The response of the cylindrical shell subjected to a normal point force.

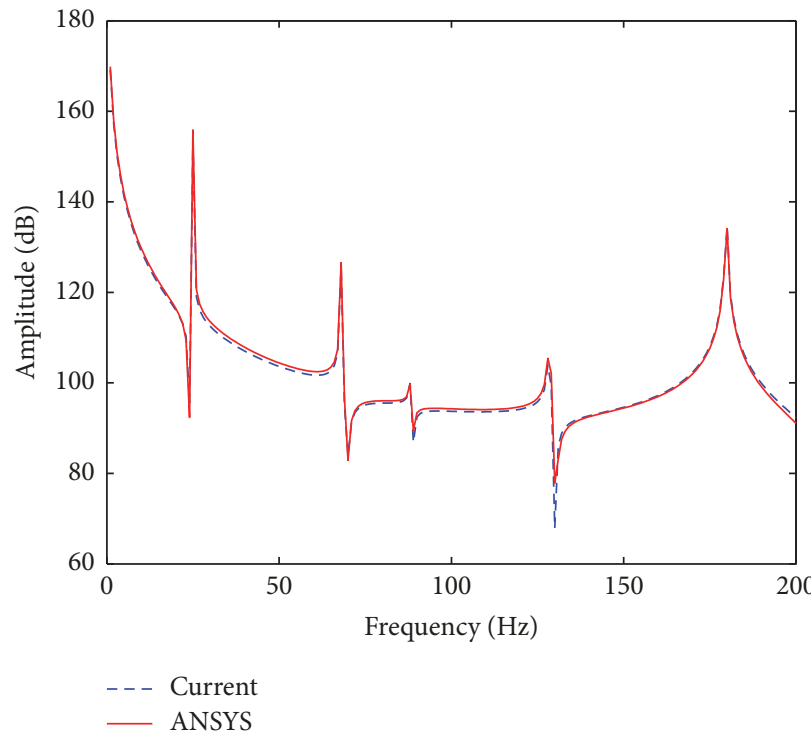

FIGURE 13: The response of the circular plate subjected to a normal point force.

change in stiffness alters only the vibration characteristics of the plate and has no effect on the shell.

\section{Conclusion}

In this paper, an improved Fourier series method (IFSM) is presented for the calculation of the vibrations of the $S$ - $P$ coupled structure with various boundary conditions. The displacement components of the cylindrical shell and circular plate are generally expanded, regardless of boundary conditions, as the superposition of a two-dimensional Fourier series and several supplementary functions. The unknown series-expansion coefficients are treated as the generalized coordinates and determined using the familiar
Rayleigh-Ritz procedure. The boundary and coupling conditions are accounted for by applying four types of translational and rotational springs with arbitrary stiffness at the junction and the boundary. Using the IFSM, the vibration characteristics of the $S$ - $P$ coupled structure with various boundary conditions and various junction positions can be obtained. The excellent accuracy of the current solutions is demonstrated by comparison with the results calculated by FEM. The response of the coupled structure and the effects of the restraint spring are illustrated.

\section{Conflicts of Interest}

The authors declare that they have no conflicts of interest. 


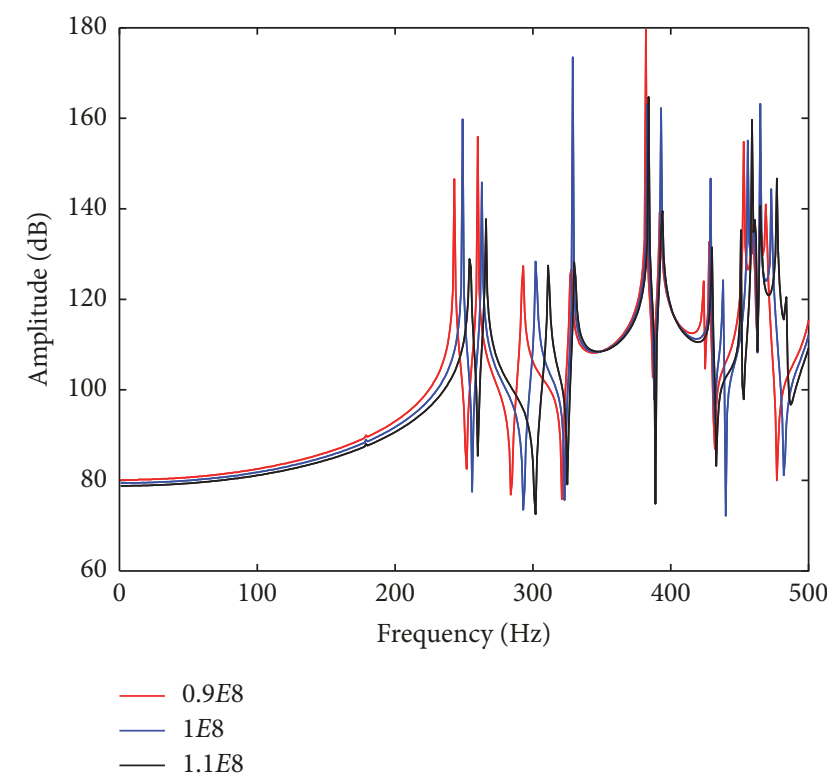

(a) $k_{v 1}$

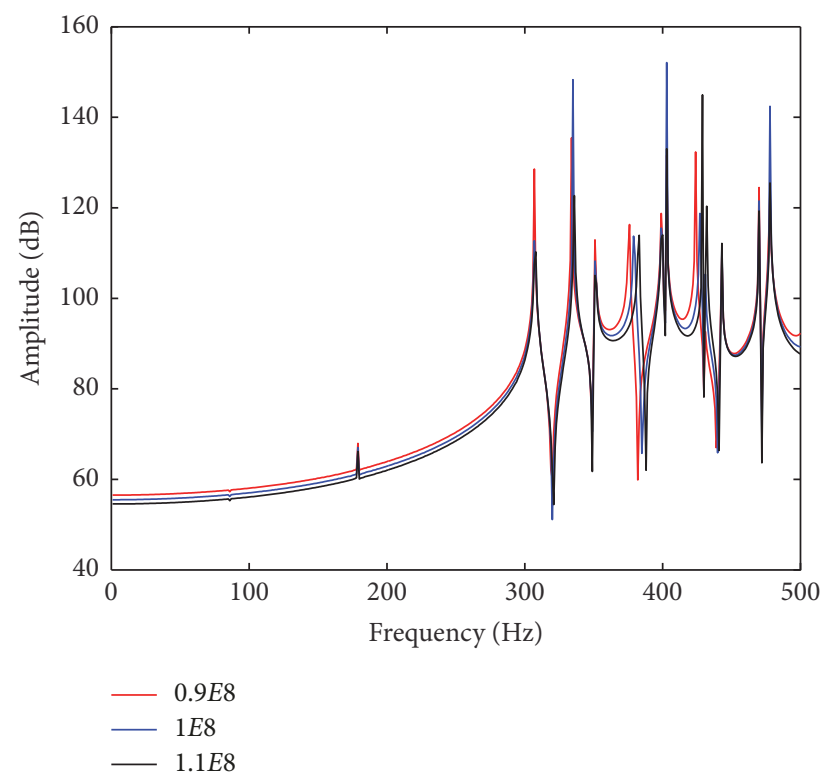

(b) $k_{w 1}$

FiguRE 14: Responses for different values of boundary-spring stiffness.

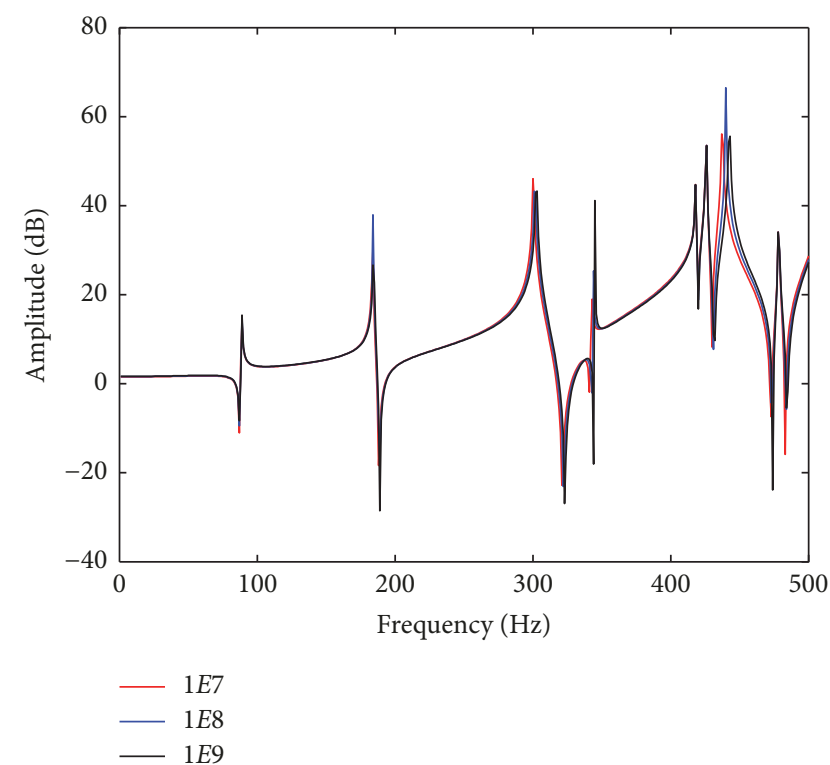

(a) The response on the plate

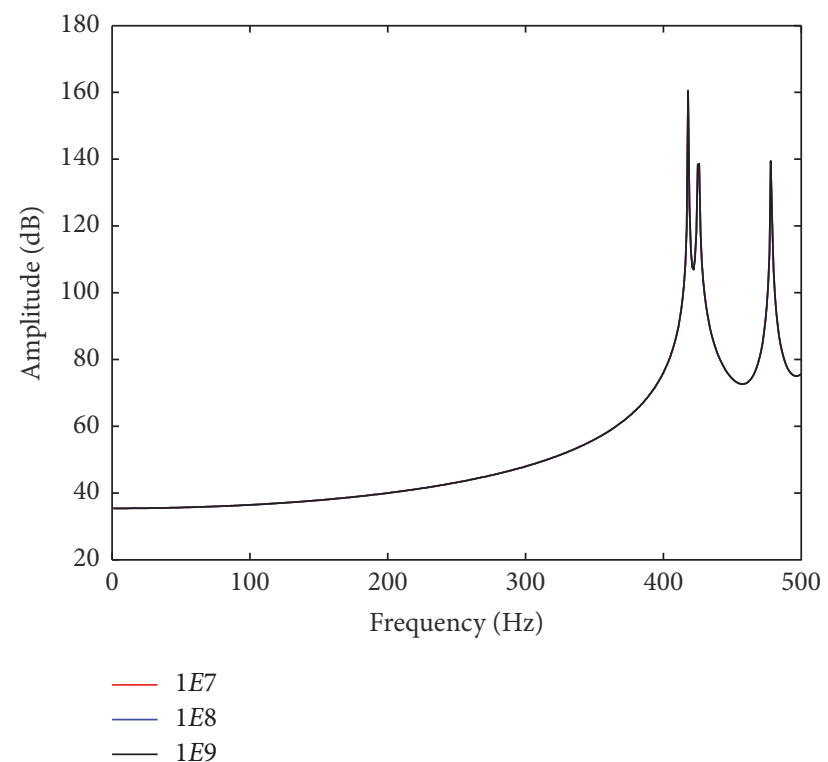

(b) The response on the shell

FIGURE 15: Responses for different values of coupling-spring stiffness.

\section{Acknowledgments}

The research is supported by the National Natural Science Foundation of China (no. 50909023 and no. 51479038). Their financial support is gratefully acknowledged.

\section{References}

[1] A. W. Leissa, Vibration of Plates, U. S. Government Printing Office, Washington, DC, USA, 1969.

[2] A. W. Leissa, Vibration of Shells, U. S. Government Printing Office, Washington, DC, USA, 1973.
[3] W. Liu, D. Wang, H. Lu, Y. Cao, and P. Zhang, "Research on radial vibration of a circular plate," Shock and Vibration, vol. 2016, Article ID 6758291, 8 pages, 2016.

[4] A. Ghoshal, S. Parthan, D. Hughes, and M. J. Schulz, "Free vibration characteristics of cylindrical shells using a wave propagation method," Shock and Vibration, vol. 8, no. 2, pp. 71$84,2001$.

[5] X. Chen and K. Ye, "Comparison study on the exact dynamic stiffness method for free vibration of thin and moderately thick circular cylindrical shells," Shock and Vibration, vol. 2016, Article ID 9748135, 14 pages, 2016. 
[6] M. S. Tavakoli and R. Singh, "Modal analysis of a hermetic can," Journal of Sound and Vibration, vol. 136, no. 1, pp. 141-145, 1990.

[7] L. Cheng and J. Nicolas, "Free vibration analysis of a cylindrical shell-circular plate system with general coupling and various boundary conditions," Journal of Sound and Vibration, vol. 155, no. 2, pp. 231-247, 1992.

[8] D. T. Huang and W. Soedel, "On the free vibrations of multiple plates welded to a cylindrical shell with special attention to mode pairs," Journal of Sound and Vibration, vol. 166, no. 2, pp. 315-339, 1993.

[9] Y. K. Tso and C. H. Hansen, "Wave propagation through cylinder/plate junctions," Journal of Sound and Vibration, vol. 186, no. 3, pp. 447-461, 1995.

[10] M. X. Chen, J. H. Wei, K. Xie, N. Q. Deng, and G. X. Hou, "Wave based method for free vibration analysis of ring stiffened cylindrical shell with intermediate large frame ribs," Shock and Vibration, vol. 20, no. 3, pp. 459-479, 2013.

[11] Y. Cao, J. Wang, and R. Zhang, "Free vibration analysis of coupled cylindrical shell-plate system with arbitrary elastic boundary conditions," in $>$ Proceedings of the 23rd International Congress on Sound and Vibration, Athens, Greece, July 2016.

[12] X. Shi, D. Shi, W. L. Li, and Q. Wang, "A unified method for free vibration analysis of circular, annular and sector plates with arbitrary boundary conditions," Journal of Vibration and Control, 2014.

[13] W. L. Li, "Free vibrations of beams with general boundary conditions," Journal of Sound and Vibration, vol. 237, no. 4, pp. 709-725, 2000. 


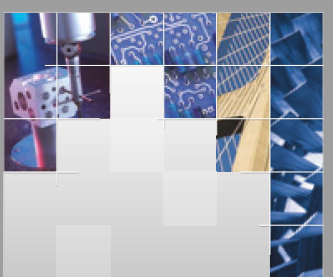

\section{Enfincering}
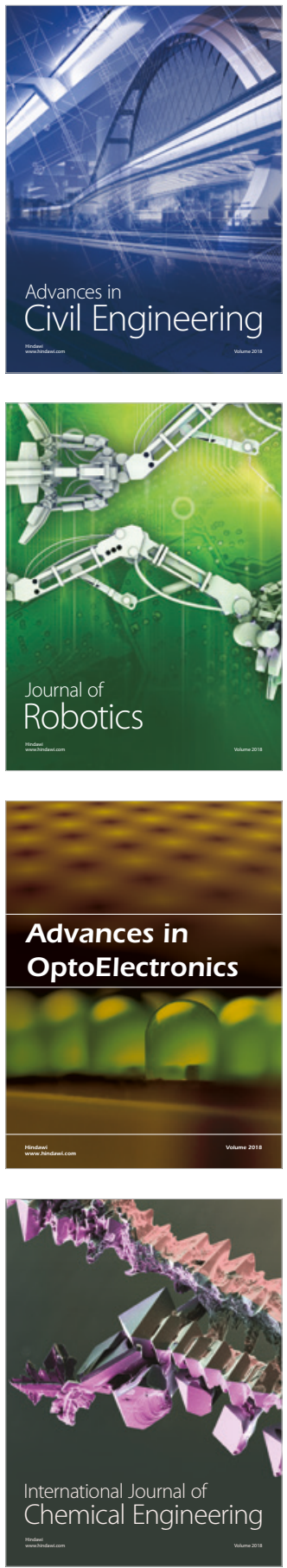

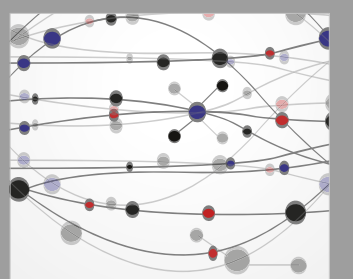

\section{Rotating \\ Machinery}

The Scientific World Journal

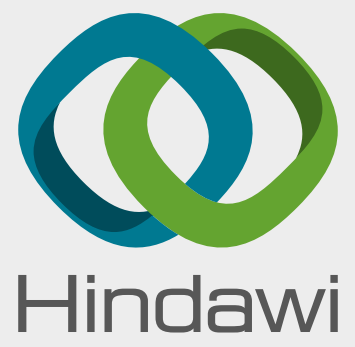

Submit your manuscripts at

www.hindawi.com
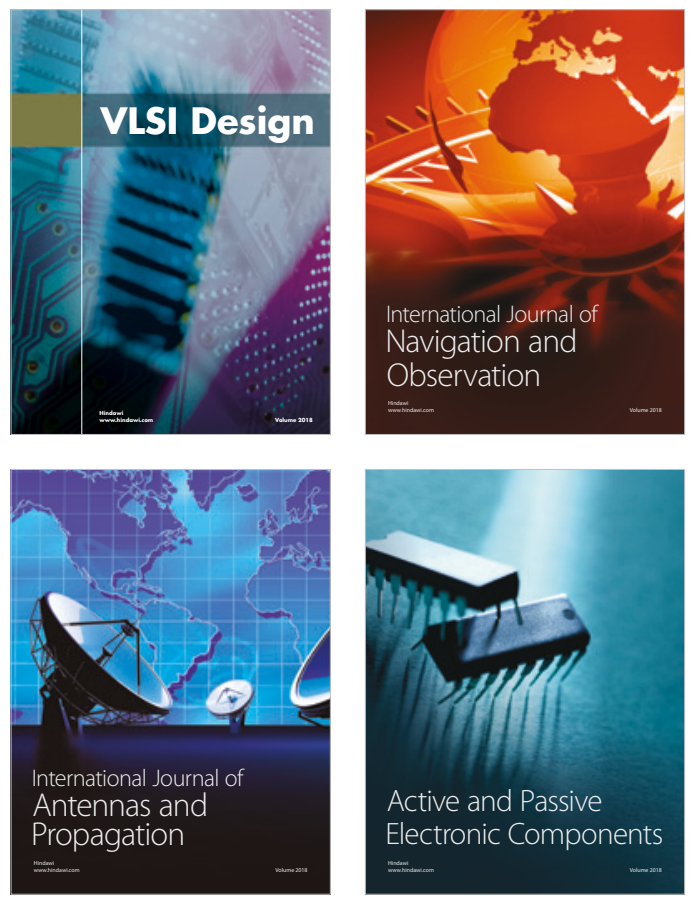
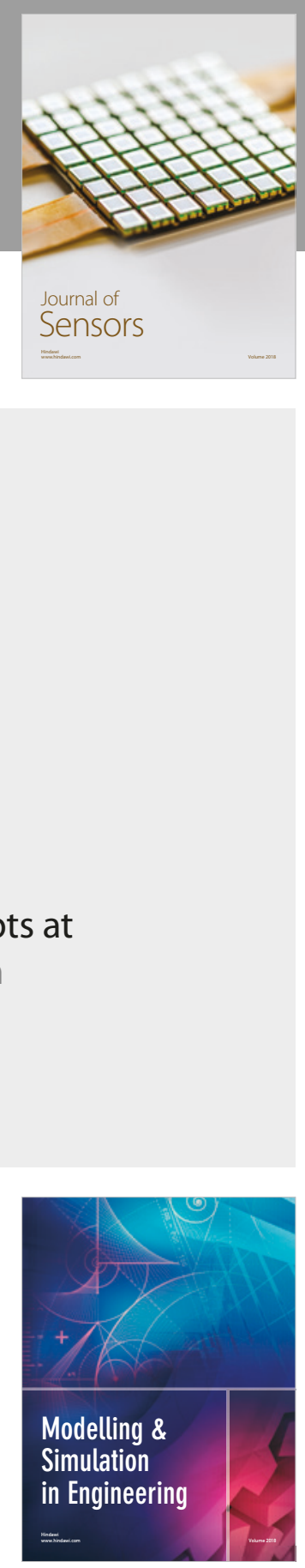

\section{Advances \\ Multimedia}
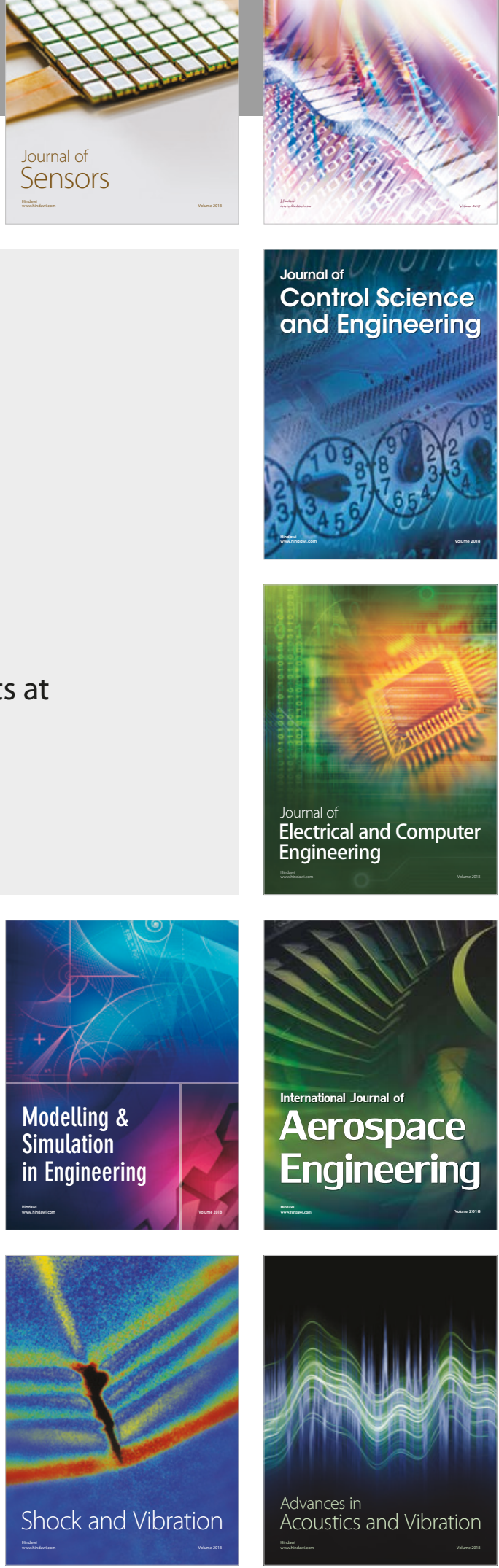